\title{
Independence number and disjoint theta graphs
}

\author{
Shinya Fujita* Colton Magnant ${ }^{\dagger}$ \\ Submitted: Aug 16, 2009; Accepted: Jul 10, 2011; Published: Jul 22, 2011 \\ Mathematics Subject Classification: 05C35
}

\begin{abstract}
The goal of this paper is to find vertex disjoint even cycles in graphs. For this purpose, define a $\theta$-graph to be a pair of vertices $u, v$ with three internally disjoint paths joining $u$ to $v$. Given an independence number $\alpha$ and a fixed integer $k$, the results contained in this paper provide sharp bounds on the order $f(k, \alpha)$ of a graph with independence number $\alpha(G) \leq \alpha$ which contains no $k$ disjoint $\theta$-graphs. Since every $\theta$-graph contains an even cycle, these results provide $k$ disjoint even cycles in graphs of order at least $f(k, \alpha)+1$. We also discuss the relationship between this problem and a generalized ramsey problem involving sets of graphs.
\end{abstract}

\section{Introduction}

The search for vertex disjoint subgraphs of a graph has been considered in many contexts. The most popular such subgraph has certainly been the cycle. Many different conditions have been established for the existence of vertex disjoint cycles (see $[1,6,12,19,24]$ ). From there, people went on to impose restrictions on the cycles. In particular, some people imposed length restrictions (see $[3,14,16]$ ), others forced cycles to contain particular vertices or edges (see $[7,13])$ while still others forced the cycles to be chorded (see $[4,10$, 17]). See [18] for a survey of degree conditions for disjoint cycles.

The structure of this paper follows that of Egawa, Enomoto, Jendrol, Ota and Schiermeyer [12]. There, the authors present many results concerning disjoint cycles in graphs with given independence number. Specifically, they define a function $g(k, \alpha)$ to be the maximum integer $n$ such that there exists a graph $G$ on $n$ vertices with independence number $\alpha(G) \leq \alpha$ and $G$ contains no $k$ disjoint cycles.

Similarly, let $g^{\prime}(k, \alpha)$ be the maximum integer $n$ such that there exists a graph $G$ on $n$ vertices with independence number $\alpha(G) \leq \alpha$ and $G$ contains no $k$ disjoint even cycles. As

*Gunma National College of Technology. 580 Toriba, Maebashi, Gunma, Japan 371-8530. Both authors were partially supported by JSPS Grant No. 20740068

${ }^{\dagger}$ Georgia Southern University, 65 Georgia Ave. Room 3008, Statesboro, GA 30460, USA.

THE ELECTRONiC JOURNAl OF COMBINATORICS 18 (2011), \#P150 
is shown in the proof of Fact 1 (see Section 2), it is easy to see that $g^{\prime}(k, \alpha) \geq 3 \alpha+4 k-4$. In $[12,19]$, it is proven that $g(k, \alpha)=3 k+2 \alpha-3$ for many cases and, in general, it seems that $g(k, \alpha)<g^{\prime}(k, \alpha)$.

In an effort to understand the function $g^{\prime}(k, \alpha)$, we use the concept of $\theta$-graphs. A $\theta$-graph is a pair of vertices with three internally disjoint paths between them. A chorded cycle is an example of a $\theta$-graph but, in general, a $\theta$-graph need not be a chorded cycle. The idea of $\theta$-graphs has been studied in a wide variety of situations (see $[2,5,8,11,15,20,22]$ ).

In particular, every $\theta$-graph contains an even cycle so, if a graph contains $k$ disjoint $\theta$-graphs, then it necessarily contains $k$ disjoint even cycles. Hence, we define another function $f(k, \alpha)$. Let $f(k, \alpha)$ be the maximum integer $n$ such that there exists a graph $G$ on $n$ vertices with $\alpha(G) \leq \alpha$ containing no $k$ disjoint $\theta$-graphs. Since $g^{\prime}(k, \alpha) \leq f(k, \alpha)$, our results provide immediate bounds $g^{\prime}(k, \alpha)$.

This research is also motivated by a ramsey-type argument. Let $\mathscr{G}$ be a class of graphs and, in particular, let $\mathscr{T}_{k}$ be the set of all possible graphs consisting of $k$ disjoint $\theta$-graphs. If we define $r(\mathscr{G}, a)$, to be the minimum integer $n$ such that any 2 coloring of $K_{n}$ results in either a copy of a graph in $\mathscr{G}$ in color 1 or a copy of $K_{a}$ in color 2 , then $f(k, \alpha)=r\left(\mathscr{T}_{k}, \alpha+1\right)-1$. Because determining ramsey numbers is extremely difficult, this analogy explains the difficulty in proving sharp bounds on $f(k, \alpha)$.

As far as the authors know, there have been no results concerning ramsey numbers for disjoint cycles versus complete graphs. The results contained in this work may be useful in the study of such problems. For example, it is clear that $r\left(\mathscr{T}_{k}, a\right) \leq r\left(k C_{2 m}, a\right)$ so a simple application of Theorem 3 provides a lower bound on the ramsey number for a collection of even cycles versus a complete graph. In trying to determine $r\left(k C_{2 m}, a\right)$ precisely, one approach may be to first show that there exist $k$ disjoint even cycles. Our result provides this first step.

The main goal of this paper is to extend the following two results. Let $\mathscr{C}_{k}$ and $\mathscr{E}_{k}$ be the sets of all graphs consisting of $k$ disjoint cycles and even cycles respectively.

Theorem 1 ([12]) For all integers $k$ and $\alpha$ with $1 \leq \alpha \leq 5$ or $1 \leq k \leq 2, r\left(\mathscr{C}_{k}, \alpha+1\right)=$ $3 k+2 \alpha-2$ (in other words, $g(k, \alpha)=3 k+2 \alpha-3)$.

More recently, Fujita managed to extend the above result to the case where $k=3$. Not surprisingly, this modest extension involved a great deal of work.

Theorem $2([19])$ For all $\alpha, r\left(\mathscr{C}_{3}, \alpha+1\right)=7+2 \alpha($ in other words, $g(3, \alpha)=6+2 \alpha)$.

Our extension is stated as follows.

Theorem 3 (Main result) For all positive integers $k$ and $\alpha$ with either $k \leq 3$ or $\alpha \leq 5$, we have $r\left(\mathscr{T}_{k}, \alpha+1\right)=3 \alpha+4 k-3$ (in other words, $\left.f(k, \alpha)=3 \alpha+4 k-4\right)$.

Somewhat surprisingly, this shows the equality $r\left(\mathscr{T}_{k}, \alpha\right)=r\left(\mathscr{E}_{k}, \alpha\right)$ (or in other words $\left.g^{\prime}(k, \alpha)=f(k, \alpha)\right)$ in many cases. Since $\mathscr{T}_{k}$ and $\mathscr{E}_{k}$ are very different sets, one may be inclined to expect the above equation to fail in general. As a result of this dilemma, we pose the following question which asks whether or not this equality always holds. 
Question 1 Is $r\left(\mathscr{T}_{k}, \alpha\right)=r\left(\mathscr{E}_{k}, \alpha\right)$ (similarly $g^{\prime}(k, \alpha)=f(k, \alpha)$ ) for all $k, \alpha$ ?

Furthermore, we extend the following results, also from [12], to $\theta$-graphs (see Section 4).

Theorem 4 ([12]) For all integers $k \geq 3$ and $\alpha \geq 6, r\left(\mathscr{C}_{k}, \alpha+1\right) \leq k \alpha$ (similarly $g(k, \alpha) \leq k \alpha+1)$.

With the addition of a minimum degree condition, the picture is very differnt. Define $g(k, \alpha, \delta)$ to be the maximum order of a graph $G$ with independence number $\alpha(G) \leq \alpha$, minimum degree $\delta(G) \geq \delta$ and no $k$ disjoint cycles.

Theorem 5 ([12]) For all integers $\alpha \geq 1, g(3, \alpha, 4) \leq 2 \alpha+6$.

In light of Theorems 1, 2 and 3, one might guess that $r\left(\mathscr{C}_{k}, \alpha+1\right)=3 k+2 \alpha-2$ and $r\left(\mathscr{T}_{k}, \alpha+1\right)=3 \alpha+4 k-3$ for all $k$ and $\alpha$. However, the following results show that this intuition is not true.

Theorem 6 ([12]) For any $c>0$, there exist $k$ and $\alpha$ such that $r\left(\mathscr{C}_{k}, \alpha+1\right)>c(k+\alpha)+1$ (similarly $g(k, \alpha)>c(k+\alpha))$.

If a graph does not contains $k$ disjoint cycles, then certainly it does not contain $k$ disjoint $\theta$-graphs so the following corollary is immediate.

Corollary 7 For any $c>0$, there exist $k$ and $\alpha$ such that $r\left(\mathscr{T}_{k}, \alpha+1\right)>c(k+\alpha)+1$ (similarly $f(k, \alpha)>c(k+\alpha))$.

In light of Corollary 7 , we state this challenging question.

Question 2 What are the minimum values of $k$ and $\alpha$ such that $f(k, \alpha)>3 \alpha+4 k-4$ ?

\section{Preliminary Results}

Using classical ramsey numbers, Egawa et. al. prove the following upper bound on $g(k, \alpha)$. Let $r(a, b)$ denote the smallest integer $n$ such that every 2-coloring of the edges of $K_{n}$ yields a copy of $K_{a}$ in color 1 or a copy of $K_{b}$ in color 2 . We will later abuse notation by using $r(G, b)$ to denote the minimum integer $n$ such that every 2 coloring of $K_{n}$ produces a monochromatic copy of $G$ in color 1 or a $K_{b}$ in color 2 .

Theorem 8 ([12]) Given positive integers $k$ and $\alpha$,

$$
g(k, \alpha) \leq 3 k+r(3, \alpha+1)-4 \leq 3 k+\frac{\alpha^{2}+2 \alpha-4}{2} .
$$

Using the same technique, we observe the following. 
Theorem 9 For all integers $k$ and $\alpha$,

$$
f(k, \alpha) \leq 4 k+r(4, \alpha+1)-5 \leq 4 k+\frac{\alpha^{3}+6 \alpha^{2}+11 \alpha}{6}-4 .
$$

Sketch of the proof: This result is proven by a simple induction on $k$. The base case is clear so suppose the result holds for values less than $k$. Consider a graph $G$ of order $4 k+r(4, \alpha+1)-4 \geq r(4, \alpha+1)$ with $\alpha(G) \leq \alpha$. Since there is no $(\alpha+1)$-independent set, there must exist a $K_{4}$ in $G$, which contains a $\theta$-graph. We may then remove the $K_{4}$ from $G$ and apply induction on $k$.

In [21] the following useful results are proven. We use these results to provide helpful structure in some of our proofs.

Theorem 10 ([21] Problem 8.20) An $\alpha$-critical graph $G$ with no isolated vertices satisfies $|G| \geq 2 \alpha(G)$.

Theorem 11 ([21] Problem 8.19) Let $G_{1}, G_{2}$ be connected $\alpha$-critical graphs other than $K_{2}$. Split a point in $G_{1}$ into two non-isolated points $x_{1}$ and $x_{2}$, remove an edge $y_{1} y_{2}$ from $G_{2}$ and identify $x_{i}$ and $y_{i}$ for $i=1,2$. The resulting graph is $\alpha$-critical and furthermore, every connected but not 3 -connected $\alpha$-critical graph arises this way.

Theorem 12 ([21] Problem 8.25) Let $G$ be a connected $\alpha$-critical graph with $|G| \geq$ $2 \alpha(G)+i$. Then the following holds:

1. If $i=0$, then $G=K_{2}$.

2. If $i=1$, then $G$ is an odd cycle with no chord.

3. If $i=2$, then $G$ is a subdivision of $K_{4}$.

Given two sets of vertices $A$ and $B$, let $e(A, B)$ be the number of edges between the sets. For a given subgraph or set of vertices $H$, define $N_{H}(v)$ to be the neighborhood of $v$ in $H$. Also let $d_{H}(v)=\left|N_{H}(v)\right|$ be the degree of $v$ in $H$. Let $\langle H\rangle$ denote the subgraph induced by the set of vertices $H$. Given vertices $a$ and $b$ in a path $P$, define $\operatorname{dist}_{P}^{\prime}(a, b)$ to be the number of vertices strictly between $a$ and $b$ on the path $P$ where $\operatorname{dist}_{P}^{\prime}(a, b)=-1$ if $a=b$. All notation not defined here may be found in [9].

We first provide a lower bound on $f(k, \alpha)$. The remainder of the paper includes a variety of upper bounds.

Fact 1 Given positive integers $k$ and $\alpha, f(k, \alpha) \geq 3 \alpha+4 k-4$.

Proof: The proof of this result is by construction. Consider the graph $G_{k, \alpha}$ consisting of $k-1$ copies of $K_{7}$ and $\alpha-k+1$ copies of $K_{3}$. Certainly $\alpha(G)=\alpha$ but there are no $k$ disjoint $\theta$-graphs.

Using Theorems 10, 11 and 12, we prove the following useful proposition. 
Proposition 1 Every connected, $\alpha$-critical graph $G$ with $|G| \geq 2 \alpha(G)+2$ contains a $\theta$-graph.

Proof: By Theorem 12, if $|G|=2 \alpha+2$, then $G$ is isomorphic to a subdivision of $K_{4}$, which contains a $\theta$-graph. Hence, let $G$ be the graph of smallest order satisfying:

- $G$ is $\alpha$-critical.

- $G$ is connected.

- $G$ contains no $\theta$-graph.

- $|G|>2 \alpha(G)+2$.

Since $G$ contains no $\theta$-graph, $G$ must not be 3-connected so by Theorem 11, $G$ can be decomposed into $\alpha$-critical graphs $G_{1}$ and $G_{2}$ where $G_{i} \neq K_{2}$. Because we assumed $|G|$ is minimum, we know that $\left|G_{i}\right| \leq 2 \alpha\left(G_{i}\right)+2$. Again if $\left|G_{i}\right|=2 \alpha\left(G_{i}\right)+2$ then Theorem 12 implies $G_{i}$ is a subdivision of $K_{4}$ which contains a $\theta$-graph. This would correspond to a $\theta$-graph in $G$, a contradiction. By Theorem 10 and since $G_{i} \neq K_{2}$, we may suppose $\left|G_{i}\right|=2 \alpha\left(G_{i}\right)+1$.

By Theorem 12, $G_{i}$ must be an odd cycle for $i=1,2$. By construction, $G$ would also be an odd cycle and so $|G|=2 \alpha(G)+1$ which is a contradiction.

The corollary below follows immediately from Proposition 1.

Corollary 13 For all $\alpha \geq 1$,

$$
f(1, \alpha)=3 \alpha .
$$

The next result provides more structure which we will use in the proof of our main result (Theorem 3).

Proposition 2 For any $\alpha$, if $\alpha(G)=\alpha$ and $|G| \geq 3 \alpha+2$ then $G$ contains either a $K_{4}^{-}$ or two disjoint $\theta$-graphs.

Proof: Let $G$ be a graph of order $3 \alpha+2$ with $\alpha(G)=\alpha$, and suppose $G$ contains no 2 disjoint $\theta$-graphs and no $K_{4}^{-}$. This result is proven by induction on $\alpha$. For the base case, if $\alpha \leq 3$, we apply the following ramsey-type argument. If $\alpha=2$, then $|G|=8>7=r\left(K_{4}^{-}, 3\right)$ so $G$ must either contain an independent set of order $3>\alpha$ or a $K_{4}^{-}$. Also, if $\alpha=3$, then $|G|=11=r\left(K_{4}^{-}, 4\right)$, we again have the desired result. Hence, we may suppose $\alpha \geq 4$.

The remainder of the proof is broken into cases based on the minimum degree.

Case 1 The minimum degree satisfies $\delta(G) \leq 3$. 
If there exists a vertex $v$ with $d(v) \leq 2$, we may remove $v$ and $N(v)$ from the graph. This creates a new graph $G^{\prime}$ with $\left|G^{\prime}\right| \geq|G|-3=3(\alpha-1)+2$ and $\alpha\left(G^{\prime}\right) \leq \alpha-1$. We may then apply induction on $\alpha(G)$ to get the desired result. Hence we assume, for the remainder of this case, that $\delta(G)=3$.

Let $v$ be a vertex of degree 3 . If we contract $v$ and $N(v)$ to a single vertex $v^{\prime}$ forming a new graph $G^{\prime}$, there must exist a $\theta$-graph $T^{\prime}=K_{4}^{-}$in $G^{\prime}$ by induction on $\alpha(G)$ (since 2 disjoint $\theta$-graphs in $G^{\prime}$ would correspond to 2 disjoint $\theta$-graphs in $G$ ). Certainly $v^{\prime} \in T^{\prime}$ since otherwise $T^{\prime}$ would be a $K_{4}^{-}$in $G$. Hence $v$ is contained in a $\theta$-graph $T$ in $G$ of order at most 7. Furthermore, if $v$ is not a hub vertex of $T$, then $|T|=6$. Let $T^{+}$be the subgraph of $G$ induced on $T \cup N(v)$. Easily we have $\left|T^{+}\right| \leq 7$ (see Figure 1 for all possible cases). Note that the dashed edges and filled vertices in Classes $V$ and $V I$ are not in $T^{+}$but are in $T^{\prime}$. Also note that there may be extra edges within these structures.

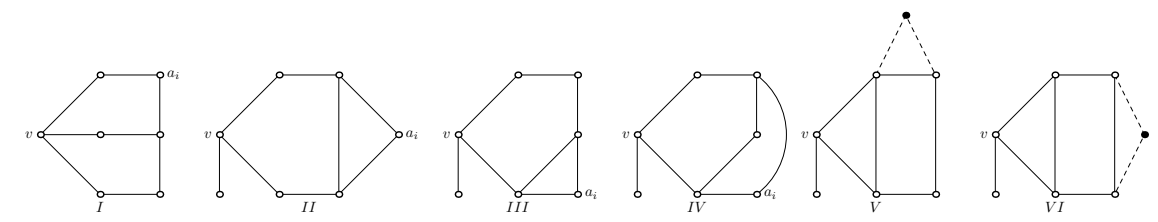

Figure 1: The possible structures of $T^{+}$.

Let $H=G \backslash T^{+}$. Since $N(v) \subseteq T^{+}$, we know $\alpha(H) \leq \alpha-1$. Conversely, if $\alpha(H) \leq$ $\alpha-2$, then $|H| \geq 3 \alpha(H)+1$ and, by Proposition $1, H$ must contain a $\theta$-graph. Hence, $\alpha(H)=\alpha-1$. Since $6 \leq\left|T^{+}\right| \leq 7$, we know $3 \alpha-4 \geq|H| \geq 3 \alpha-5 \geq 3 \alpha(H)-2$. Let $H^{\prime}$ be a spanning $\alpha$-critical subgraph of $H$ with the greatest number of triangles. Since $H$ is $\theta$-graph free, $H^{\prime}$ is a collection of components, each of which is an odd cycle, a $K_{1}$ or a $K_{2}$. In fact, since $|H| \geq 3 \alpha(H)-2, H^{\prime}$ is a collection of triangles with exactly one of the following classes of components:

1. $C_{7}$,

2. $K_{1}$,

3. at most two of $C_{5}$ or $K_{2}$.

Certainly if there are two of $C_{5}$ or $K_{2}$, there can be at most two edges of $H$ between these components of $H^{\prime}$. There cannot be two edges between two copies of $C_{5}$ without forming a $\theta$-graph. If there are two edges between a $C_{5}$ and $K_{2}$, since $H$ contains no $\theta$-graph, these edges must be incident to a single vertex of the $C_{5}$. In this case, we can switch these two components of $H^{\prime}$ for three components, one of which is another triangle and two are copies of $K_{2}$. This contradicts the choice of $H^{\prime}$.

The final case is when there are two edges between copies of $K_{2}$. If the two edges meet at a single vertex in one copy of $K_{2}$, we can switch $H^{\prime}$ to include a triangle and a copy of $K_{1}$, again contradicting the choice of $H^{\prime}$. Hence, two extra edges between copies of $K_{2}$ 
must form a $C_{4}$. For the sake of notation in Claim 1, we will call this a $C_{4}$ of $H^{\prime}$ (this is an abuse of notation since certainly $C_{4}$ is not $\alpha$-critical).

The following claim applies to any single component in the above classes.

Claim 1 Let $C$ be a $C_{7}, C_{5}, C_{4}, K_{1}$ or $K_{2}$ in $H^{\prime}$ (with at most one edge between copies of $C_{5}$ and / or $K_{2}$ ). Any maximum independent set of $C$ may be extended to a maximum independent set of $H$. Furthermore, for each triangle of $H^{\prime}$, there is always a choice of at least two vertices for the constructed maximum independent set and every maximum independent set of $H$ can be constructed in this way.

Proof of Claim 1: Let $C$ be a component of the above classes in $H^{\prime}$. Consider any maximum independent set $I$ of $C$. Remove $I \cup N(I)$ from $H$ and consider the remaining graph. Recall that all but at most one component of $H^{\prime} \backslash C$ must be triangles (where the single component could be either $K_{2}$ or $C_{5}$ ).

At most one vertex could have been removed from each other component of $H^{\prime}$ (since, if more than one edge goes from $C$ to a triangle, this would form a $\theta$-graph). Hence, there are at least two vertices left in each triangle. Furthermore, there is at least one vertex remaining if the component is a $K_{2}$ and at least four if the component is a $C_{5}$. Let $\tau_{1}$ be the set of components of $H^{\prime}$ missing a vertex.

Choose one vertex from each component of $\tau_{1}$. Note that these vertices must be independent in $H$ or else there would be a $\theta$-graph in $H$. Add these vertices to $I$ creating a new independent set $I_{1}$. We remove all vertices in $I_{1} \cup N\left(I_{1}\right)$ from the graph and proceed creating sets $\tau_{2}$ and $I_{2}$.

This step is repeated to generate a large independent set. If, at any point $\tau_{i}$ is empty, arbitrarily choose a remaining component from $H^{\prime}$ for $\tau_{i}$ and continue the process. Since $H$ contains no $\theta$-graph, this process terminates at a maximum independent set of $H$. Note that, at every step, we have a choice of at least two vertices for each triangle.

In order to show that every maximum independent set of $H$ can be constructed in this manner, we need only notice that any independent set contains at most one vertex of each triangle. Hence, every maximum independent set of $H$ must contain a maximum independent set of the classes given in the statement. This completes the proof of the claim.

Let $A=V\left(T^{+}\right) \backslash(\{v\} \cup N(v))$. These vertices are chosen for their potential to be in an independent set with $v$. Since $\left|T^{+}\right| \geq 6$ we know that $|A| \geq 2$. In fact, since $T^{+}$must look like one of the graphs in Figure 1, we note that, except in Classes $V$ and $V I$, the set $A$ contains a $P_{3}$ and, in every case, $A$ is connected. Label the vertices of such a $P_{3}$ with $a_{1}, a_{2}, a_{3}$ in order (in Classes $V$ and $V I$, label the vertices with $a_{1}$ and $a_{2}$ arbitrarily).

If there exists a maximum independent set $I$ of $H$ for which $e\left(a_{i}, I\right)=0$ for some $a_{i} \in$ $A$, then $I \cup\left\{a_{i}, v\right\}$ is an independent set of order $\alpha(H)+2>\alpha$, which is a contradiction. Hence, $a_{i}$ must be adjacent to at least one vertex in every maximum independent set of $H$ for all $i \in\{1,2,3\}$.

Conversely, since $G$ does not contain a $K_{4}^{-}$, no vertex of $A$ may be adjacent to more than one vertex of a triangle in $H$. Let $\mathscr{C}$ be the set of non-triangle components of $H^{\prime}$. 
By Claim 1, there is a choice of at least two vertices in each triangle for any maximum independent set extended from a maximum independent set of a component $C \subseteq \mathscr{C}$. This means that, in order to be adjacent to a vertex of every maximum independent set of $H$, each vertex of $A$ must be adjacent to a vertex in every maximum independent set of at least one component of $\mathscr{C}$. This provides a pairing (not necessarily unique) of the vertices in $A$ to the components of $\mathscr{C}$.

When a vertex $a_{i}$ is paired with a component $C$ where $C$ is an odd cycle, we use the fact that, in any odd cycle, for any choice of two vertices, there exists a maximum independent set of the cycle avoiding these two vertices. In order for $a_{i}$ to be adjacent to at least one vertex of every maximum independent set of $C$, the vertex $a_{i}$ must be adjacent to at least 3 vertices of $C$. Also note that, since $G$ is $K_{4}^{-}$-free, $a_{i}$ cannot be adjacent to 3 consecutive vertices of $C$.

The remainder of the proof of this case consists of considering cases based on the structure of $\mathscr{C}$.

First suppose $C=C_{7}$. This means that $|H|=3 \alpha(H)-2$ which implies $\left|T^{+}\right|=7$ and hence $|A|=3$, meaning that we have only classes I through IV. If we label the vertices of the cycle with $u_{1}, u_{2}, \ldots, u_{7}$ in order, each vertex in $A$ must be adjacent to $u_{1}, u_{2}$ and $u_{5}$ or some rotation of this (otherwise $a_{1}$ cannot be adjacent to a vertex of every maximum independent set of $C$ ). Without loss of generality, suppose $a_{1}$ is adjacent to $u_{1}, u_{2}$ and $u_{5}$. The vertex $a_{2}$ cannot be adjacent to $u_{1}$ or $u_{2}$ without forming a $K_{4}^{-}$. Hence, the adjacencies of $a_{2}$ must be (up to symmetry) $u_{3}, u_{4}$ and $u_{7}$. By a similar argument, we find that $a_{3}$ must be adjacent to $u_{5}, u_{6}$ and $u_{2}$.

For any vertex $a_{i}$, there exists a segment between two neighbors of $a_{i}$ on $C$ which contains at least one neighbor of each $a_{j}$ for $j \neq i$. This means that for any vertex $a_{i}$, we can construct a $\theta$-graph using $a_{i} \cup C$ and still use a segment of $C$ to find an extra path between the vertices of $A \backslash a_{i}$.

In every case from Figure 1 (except classes $V$ and $V I$ ), there exists a vertex in $A$ (labeled $a_{i}$ ) such that, if we remove $a_{i}$ from $T^{+}$but provide another path between the vertices of $A \backslash a_{i}$, the result will still contain a $\theta$-graph. As above, this vertex $a_{i}$ may be used to construct another $\theta$-graph using $C$. This process creates two disjoint $\theta$-graphs which is a contradiction.

Next we suppose $C=K_{1}$ and call this vertex $u$. This implies that $|A|=3$ and all of $A$ is adjacent to $u$. Since $A$ forms a path within $T^{+}$, we see that $A \cup\{u\}$ forms a $K_{4}^{-}$, a contradiction.

Next, suppose there exist two of $C_{5}$ or $K_{2}$ in $\mathscr{C}$ implying that $|A|=3$, and $\left|T^{+}\right|=7$. Further, we suppose there is at most one edge of $H$ between these components of $H^{\prime}$. Note that at most one vertex of $A$ may be paired with a copy of $K_{2}$ in order to avoid a $K_{4}^{-}$. Also, the only way for a vertex $a_{j} \in A$ to be adjacent to a vertex of every maximum independent set of a $C_{5}$ is if $a_{j}$ is adjacent to 3 consecutive vertices of the cycle. This creates a $K_{4}^{-}$which is a contradiction. Hence, there can be at most two vertices of $A$ paired with components of $\mathscr{C}$ (if both are isomorphic to $K_{2}$ ) but since $|A|=3$, this is a contradiction. 
If there are two edges of $H$ between copies of $C_{5}$ or $K_{2}$, by the choice of $H^{\prime}$, this structure must form a $C_{4}$. Label the vertices of the $C_{4}$ with $v_{1}, v_{2}, v_{3}, v_{4}$. Recall that the three vertices of $A$ induce at least a path. Also recall the above labeling of the vertices in $A$ with $a_{1}, a_{2}, a_{3}$.

Note that there are two disjoint maximum independent sets of a $C_{4}$. Since each vertex of $A$ must be adjacent to a vertex of every maximum independent set of this $C_{4}$, each vertex of $A$ must be adjacent to a pair of consecutive vertices of the $C_{4}$. Without loss of generality, suppose $a_{1}$ is adjacent to $v_{1}$ and $v_{2}$. If $a_{2}$ shares even one adjacency with $a_{1}$ (suppose $a_{2}$ is adjacent to $v_{2}$ and $v_{3}$ ) then the set $\left\{a_{1}, a_{2}, v_{1}, v_{2}\right\}$ induces a $K_{4}^{-}$in $G$, a contradiction. Hence, $a_{2}$ must be adjacent to $v_{3}$ and $v_{4}$. Finally, by the same argument, $a_{3}$ must not share any adjacencies with $a_{2}$ so $a_{3}$ must be adjacent to $v_{1}$ and $v_{2}$. This time, the set $\left\{a_{1}, a_{2}, v_{1}, v_{2}\right\}$ induces a $K_{4}^{-}$, which is a contradiction.

Finally, we suppose there exists only one of $C_{5}$ or $K_{2}$. In this case, $|H|=3 \alpha(H)-1$. Still, $|A| \geq 2$ but, as above, no vertex may be paired with a $C_{5}$. Since only one vertex of $A$ may be paired with a copy of $K_{2}$, we arrive at a contradiction, completing the proof of this case.

Case 2 The minimum degree satisfies $\delta(G) \geq 4$.

We first show that there exists a $\theta$-graph of order 5 . Since $f(1, \alpha)+1=3 \alpha+1<$ $3 \alpha+2=|G|$, we know there exists a $\theta$-graph $T$ and there exists at least one vertex $v \in H=G \backslash T$. Choose $T$ such that $|T|$ is minimum and suppose $|T| \geq 6$. If $d_{T}(v) \geq 3$ we can use $v$ to make $|T|$ smaller. Hence $d_{T}(v) \leq 2$ for all $v \in H$, so $\delta(H) \geq 2$.

If there exists a pair of adjacent vertices $v_{1}, v_{2} \in H$ with $d_{T}\left(v_{i}\right) \geq 2$, then, since $|T| \geq 6$, we may again make $|T|$ smaller. This implies that, for every vertex $v$ of degree 2 in $H$, every vertex $u \in N(v) \cap H$ must have $d_{T}(u) \leq 1$ so $d_{H}(u) \geq 3$.

Consider the graph $H^{*}$ constructed by reducing every vertex of degree 2 in $H$ to a single edge. Certainly $\delta\left(H^{*}\right) \geq 3$ so it must contain a $\theta$-graph. This corresponds to a $\theta$-graph in $H$ which is a contradiction. Hence, we may suppose $|T|=5$.

Since $\alpha \geq 4$ we get the following useful claim.

Claim 2 There exist two vertex disjoint cycles in $H$.

Proof of Claim 2: $\quad$ Suppose $H$ does not have two vertex disjoint cycles. We first observe an easy fact about $H$.

Fact 2 Let $H$ be a graph with no $\theta$-graph and no two disjoint cycles. Then there is a vertex $v \in H$ such that $H \backslash\{v\}$ is a forest.

For the proof of this fact, we may certainly assume that $H$ contains a cycle. If $H$ contains a single cycle, any vertex on the cycle would suffice. If $H$ contains more than one cycle, they must all share a single vertex $v$ in order to avoid constructing either a $\theta$-graph or two disjoint cycles. The removal of $v$ destroys all cycles in $H$, leaving behind a forest. 
By Fact 2 , if $\alpha \geq 5$, then it follows that $\alpha(H) \geq\lceil(|H|-1) / 2\rceil=\lceil(3 \alpha-4) / 2\rceil>\alpha$, a contradiction. Hence, we may assume that $\alpha=4$ and $H$ contains an odd cycle $C$. Let $v$ be the vertex which is contained in every cycle of $H$ (from Fact 2). Let $H_{1}, \ldots, H_{\ell}$ be the components of $H \backslash\{v\}$. Since $\alpha(H)=4$, we see that $\ell \leq 4$.

Fact 3 For any edge $e=x y$ in $H, \min \{e(x, T), e(y, T)\} \leq 2$.

This fact follows easily from the observation that if both end vertices of this edge had at least three edges to $T$, there must exist a $K_{4}^{-}$in $T \cup e$. The remainder of the proof proceeds by proving the following claims.

Subclaim $1 e(H \backslash\{v\}, T) \geq 16$.

Proof of Subclaim 1: Since $H$ contains no $\theta$-graph, for each $1 \leq i \leq \ell$, we have $e\left(v, H_{i}\right) \leq 2$. It follows from the assumption $\delta(G) \geq 4$ that for each $i$ with $1 \leq i \leq \ell$, we get

$$
\begin{aligned}
4\left|H_{i}\right| & \leq \sum{ }_{x \in H_{i}} d_{G}(x) \\
& =\sum d_{G-H_{i}}(x)+\sum d_{H_{i}}(x) \\
& =\sum d_{G-H_{i}}(x)+2\left|E\left(H_{i}\right)\right| \\
& =\sum d_{G-H_{i}}(x)+2\left|H_{i}\right|-2,
\end{aligned}
$$

and hence

$$
2\left|H_{i}\right|+2 \leq \sum d_{G-H_{i}}(x)=e\left(H_{i}, T\right)+e\left(H_{i}, v\right) \leq e\left(H_{i}, T\right)+2 .
$$

Consequently, from the fact that $|H|=9$, we have

$$
16=2 \sum_{i=1}^{\ell}\left|H_{i}\right| \leq \sum_{i=1}^{\ell} e\left(H_{i}, T\right)=e(H \backslash\{v\}, T)
$$

which is the desired inequality.

Subclaim 1

Subclaim $2 H$ is connected.

Proof of Subclaim 2: For a contradiction, suppose that $H_{1}$ is a component of $H \backslash\{v\}$ with $e\left(H_{1}, v\right)=0$. By Fact 3 and the assumption that $\delta(G) \geq 4$, it is easy to see that $\left|H_{1}\right| \geq 3$. Recall that, by definition, $H_{1}$ is a tree. Hence, there exist vertices $v_{1}, v_{2} \in H_{1}$ with $v_{1} v_{2} \notin E(G)$ such that $e\left(v_{j}, T\right) \geq 3$ for $j=1,2$.

Since $G$ does not contain a $K_{4}^{-}$, this implies that $T \cong K_{2,3}$ and, when we let $A, B$ be partite sets of the $K_{2,3}$ with $|A|=2,|B|=3$, we see that $A \cup\left\{v_{1}, v_{2}\right\}$ forms an independent set. Moreover, for each $x \in A$, the graph $\left\langle(T \backslash\{x\}) \cup H_{1}\right\rangle$ contains a $\theta$-graph. Also, since $\alpha(G)=4$, note that for each $y \in H \backslash H_{1}, e(y, A)>0$. Now, consider a cycle $C$ in $H \backslash H_{1}$. By the above observation, we see that there is a vertex $x \in A$ such that $\langle C \cup\{x\}\rangle$ forms a $\theta$-graph. This allows us to find two disjoint $\theta$-graphs, a contradiction.

$\square$ Subclaim 2 
Subclaim $3 \delta(H) \geq 2$.

Proof of Subclaim 3: Suppose not. By Subclaim 2, we have $\delta(H)=1$. Take a vertex $x \in H$ such that $d_{H}(x)=1$. Then, it follows that $3 \leq e(x, T) \leq 5$. It is easy to check that for any $y \in T,\langle(T \backslash\{y\}) \cup\{x\}\rangle$ contains a $\theta$-graph. By Subclaim $2, H$ is connected and so $H \backslash x$ is also connected. If a vertex $y \in T$ had 3 edges to $H \backslash x$, this would form a second (vertex disjoint) $\theta$-graph, a contradiction. Therefore, $e(y, H \backslash\{x\}) \leq 2$ for all $y \in T$. However, this contradicts Subclaim 1 completing the proof of this claim.

$\square_{\text {Subclaim } 3}$

In view of Subclaims 2 and 3 , we see that each $H_{i}$ is a path and each endvertex of the path is adjacent to $v$. Hence, since $H$ contains no $\theta$-graph, no internal vertex of any path is adjacent to $v$. This means we only have to check cases based on the value of $\ell$.

First we consider the case $\ell=1$ which implies $H \cong C_{9}$. Since there are at least 18 edges between $H$ and $T$ (by the degree of the vertices in $H$ ), there is a vertex $u \in T$ such that $e(u, H) \geq 4$. Since $G$ does not contain a $K_{4}^{-}$, we see that $e(u, H) \leq 6$. Then, take a shortest segment $I$ in $H$ (along the cycle) such that $\langle I \cup u\rangle$ forms a $\theta$-graph. Note that both $T \backslash\{u\}$ and $H \backslash I$ are connected. In order to avoid another $\theta$-graph, $e(T \backslash\{u\}, H \backslash I) \leq 2$, which easily leads to a contradiction.

If $\ell=2$, then $H$ is either constructed by identifying a vertex of two copies of $C_{5}$ or a $C_{7}$ and a triangle. Recall that $H$ contains an odd cycle so we need not consider the case where $H$ is constructed from $C_{4}$ and $C_{6}$. First suppose $H$ is constructed from a $C_{7}$ (call it $C$ ) and a triangle. Let $P=C \backslash\{v\}$ and note that each vertex of $P$ must have two edges to $T$ since $\delta(G) \geq 4$. This implies that there exists a vertex $u \in T$ with three edges to $P$, and $P \cup\{u\}$ forms a $\theta$-graph. Now note that each vertex of the triangle (except $v$ ) also has at least 2 edges to $T$. Hence, there are two edges from the triangle to $T \backslash\{u\}$ which do not share a vertex in the triangle. This forms a second $\theta$-graph, a contradiction.

Hence, we suppose $H$ can be constructed by identifying a vertex in two 5 -cycles $C_{1}$ and $C_{2}$. Label the vertices of $C_{i}$ with $v_{i, 1}, v_{i, 2}, \ldots, v_{i, 5}$ so that $v_{1,5}=v_{2,5}=v$. First suppose there exists a vertex $u \in T$ with edges to $v_{i, 1}$ and $v_{i, 4}$ for each $i$ (and not $v_{i, 2}$ or $v_{i, 3}$ for either $i$ ). Then $\{u, v\} \cup N_{H}(u)$ forms a $\theta$-graph. Now notice that $v_{1,2}$ and $v_{1,3}$ both have two edges to $T \backslash u$ and this forms a second $\theta$-graph, a contradiction. Hence, in order to avoid creating an independent set of size 5, every vertex of $T$ must be adjacent to either $v_{i, 1}$ and $v_{i, 2}$ or $v_{i, 3}$ and $v_{i, 4}$ for some $i$. Since there are 5 vertices in $T$ and only 4 options for pairs of neighbors, there must be at least two vertices in $T$ which share such a pair of neighbors in $H$. This forms a $K_{4}^{-}$, a contradiction.

When $\ell=3, H$ is constructed by identifying a single vertex in each of two triangles and a 5-cycle. This time we need not consider the case where $H$ is constructed from a triangle and two copies of $C_{4}$ because the independence number of that graph is 5 , a contradiction. Let $C$ be the 5 -cycle and label the vertices of $C$ with $v_{1}, v_{2}, v_{3}, v_{4}$ and $v_{5}=v$. In order to avoid creating an independent set of size 5 without creating a $K_{4}^{-}$, each vertex $u \in T$ must be adjacent to either $v_{1}$ and $v_{2}$ or $v_{3}$ and $v_{4}$ or $v_{1}$ and $v_{4}$. In order to avoid a $K_{4}^{-}$, we see that $T=K_{2,3}$ and the each vertex of the 3 -set are adjacent to $v_{1}$ 
and $v_{4}$. If we let $u$ be a vertex in the 2 -set, each of the above possibilities for adjacencies in $C$ results in a $K_{4}^{-}$, a contradiction.

Finally, when $\ell=4$, it follows that $H \cong K_{1}+4 K_{2}$. In this case, note that for each vertex $y \in T$, there exists an independent set $I$ of size 4 in $H$ such that $e(y, I)=0$ because $G$ does not contain $K_{4}^{-}$. However, this contradicts $\alpha(G)=4$.

Claim 2

Let $C_{1}$ and $C_{2}$ be two disjoint cycles in $H$. If these two cycles are in the same component of $H$, there exists a path $P$ with exactly one end-vertex in each cycle. Because every cycle has order at least 3 , there must exist two vertices on each cycle which are not endpoints of $P$. Consider vertices $u_{i, 1}, u_{i, 2} \in C_{i} \backslash P$ (if such a path $P$ exists; otherwise choose any vertices of $C_{i}$ ). For each vertex $u_{i, j}$, if $d_{H}\left(u_{i, j}\right)=2$, then we call this vertex $v_{i, j}$. If not, then there is a path from $u_{i, j}$ to a leaf, which we call $v_{i, j}$, with $d_{H}\left(v_{i, j}\right)=1$. In either case, we have two vertices for each cycle $C_{i}$, which have degree, into $T$, at least 2 .

The reader may verify that, no matter how these edges fall into $T$, we can decompose $T$, using the vertices $v_{i, j}$ and their associated cycles, to create two disjoint $\theta$-graphs in $G$. This completes the proof of Proposition 2.

The corollary below follows almost immediately from Proposition 2.

Corollary 14 For all $\alpha \geq 1$,

$$
f(2, \alpha)=3 \alpha+4
$$

Proof: Let $G$ be a graph of order $3 \alpha+5$ with $\alpha(G)=\alpha$ and suppose there are no two disjoint $\theta$-graphs. By Proposition 2 , there exists a $K_{4}^{-}$in $G$. This means that $|G \backslash T|=|G|-4=3 \alpha+1$. Since $f(1, \alpha)=3 \alpha$ and $\alpha(G \backslash T) \leq \alpha(G)$, there exists another $\theta$-graph in $H$.

\section{Proof of our Main Result}

Our main result shows that Fact 1 is, in fact, sharp for many small values of $k$ and $\alpha$.

Theorem 3 Given a positive integers $k$ and $\alpha$ such that either $k \leq 3$ or $\alpha \leq 5, f(k, \alpha)=$ $3 \alpha+4 k-4$.

Proof: The lower bound follows from Fact 1 . When $k=1,2$, Corollaries 13 and 14 respectively imply that $f(k, \alpha)=3 \alpha+4 k-4$.

Suppose $k \geq 3$ and $\alpha \leq 5$. By Proposition 2 and by induction on $k$, we may assume

$G$ contains no $K_{4}^{-}$. Therefore we may use known ramsey numbers as follows. Since $\alpha(G)=\alpha$, we may also assume there is no clique of size $\alpha+1 \mathrm{in} \bar{G}$. The following table 
of orders of $G$ relative to known ramsey numbers (from [23]) takes care of all cases when $\alpha \leq 5$.

\begin{tabular}{c|c|c}
$\alpha$ & $|G| \geq$ & $r\left(K_{4}^{-}, \alpha+1\right)$ \\
\hline 2 & 15 & 7 \\
3 & 18 & 11 \\
4 & 21 & 16 \\
5 & 24 & 21
\end{tabular}

Finally we suppose $k=3$ and $\alpha \geq 6$. If $\delta(G) \leq 2$, we may remove a vertex of degree 2 and its neighbors to make a new graph $G^{\prime}$ with $\left|G^{\prime}\right| \geq|G|-3$ and $\alpha\left(G^{\prime}\right) \leq \alpha(G)-1$ and proceed by induction on $\alpha$.

If $\delta(G)=3$, let $v$ be a vertex with $d(v)=3$. Since $G$ contains no copy of $K_{4}^{-}$, there exists a pair of vertices $u, u^{\prime} \in N(v)$ such that $u u^{\prime} \notin E(G)$. Contract $v$ and $N(v)$ to a single vertex $v^{\prime}$ and thereby construct a new graph $G^{\prime}$ with $\left|G^{\prime}\right|=|G|-3$. We claim that $\alpha\left(G^{\prime}\right)<\alpha(G)$. Let $I^{\prime}$ be a maximum independent set of $G^{\prime}$ and first suppose $v^{\prime} \notin I^{\prime}$. Then the set $I=I^{\prime} \cup\{v\}$ is an independent set of $G$ that is larger than $I^{\prime}$. Finally, suppose $v^{\prime} \in I^{\prime}$. Then the set $I=\left(I^{\prime} \backslash v^{\prime}\right) \cup\left\{u, u^{\prime}\right\}$ forms a larger independent set in $G$. This means that $\alpha\left(G^{\prime}\right)<\alpha(G)$ and we may then apply induction on $\alpha$ to find three disjoint $\theta$-graphs in $G^{\prime}$ which correspond to the same in $G$, a contradiction. Hence, we assume that $\delta(G) \geq 4$.

Recall that our goal is to show that $f(3, \alpha)=3 \alpha+8$. Let $G$ be a graph on $n=3 \alpha+9$ vertices. Since $f(2, \alpha)=3 \alpha+4$, there exist two disjoint $\theta$-graphs $T_{1}$ and $T_{2}$ in $G$. Let $V_{4}$ be the vertices of $G$ of degree 4 .

Choose such $\theta$-graphs with the following:

\section{Properties:}

1. $G \backslash\left(T_{1} \cup T_{2}\right)$ contains an edge,

2. subject to the above, $\left|T_{1} \cup T_{2}\right|$ is as small as possible,

3. subject to the above, if possible, we prefer $\theta$-graphs containing vertices of $V_{4}$.

In the following argument, we often try to replace $T_{i}$ by another $\theta$-graph. In every application of this process, we replace $T_{i}$ so that Property 1 (above) is preserved.

Let $H=G \backslash\left(T_{1} \cup T_{2}\right)$ and notice that $|H| \geq 4$. By induction on $\alpha$, we may assume there is no $K_{4}^{-}$in $G$. If $\left|T_{i}\right| \leq 7$ for some $i$, then we may apply Proposition 2 on $G \backslash T_{i}$ to find a total of three disjoint $\theta$-graphs. Hence, we may suppose $\left|T_{i}\right| \geq 8$ for each $i$.

Given a triangle $S=x y z x$ in $H$, if $d_{H}(y)=d_{H}(z)=2$ and $y, z \in V_{4}$, then $S$ is called a special triangle with a central vertex $x$. The following several claims will be used to prove the desired result.

Claim 3 Let $T$ be a $\theta$-graph in $G$. Suppose that $T$ contains a vertex $v$ such that $v \in V_{4}$. Then, the following statements hold: 
(i) $|T| \geq 9$, and the equality holds only if $v$ is not a hub vertex in $T$.

(ii) If there exists a vertex $u$ in $T$ such that $u \in V_{4}$ and $u v \notin E(G)$, then $|T| \geq 10$.

Proof of Claim 3: $\quad$ For part (i), let $T$ be a $\theta$-graph in $G$ and suppose $T$ contains a vertex $v \in V_{4}$ and $|T| \leq 8$. Consider the graph $H_{1}=G \backslash(T \cup N(v))$. Since the vertex $v$ and its entire neighborhood lies outside $H_{1}$, we find that $\alpha\left(H_{1}\right) \leq \alpha(G)-1 \leq \alpha-1$ so we get

$$
\left|H_{1}\right| \geq|G|-|T|-2 \geq|G|-10=3 \alpha-1 \geq 3 \alpha\left(H_{1}\right)+2 .
$$

By Proposition 2, there exists either a $K_{4}^{-}$in $H_{1}$ or two disjoint $\theta$-graphs. Either case results in a contradiction.

If $v$ is a hub vertex of $T$ and $|T|=9$, we find that $\left|H_{1}\right| \geq|G|-|T|-1 \geq|G|-10$ and the same argument provides a contradiction.

For part (ii), we again let $T$ be a $\theta$-graph of order at most 9 in $G$ and suppose $u, v \in T \cap V_{4}$ with $u v \notin E(G)$. Let $H_{2}=G \backslash(T \cup N(u) \cup N(v))$. This time we get $\alpha\left(H_{2}\right) \leq \alpha-2$ so

$$
\left|H_{2}\right| \geq|G|-|T|-4 \geq|G|-13=3 \alpha-4 \geq 3 \alpha\left(H_{2}\right)+2
$$

and we may again apply Proposition 2 on $\mathrm{H}_{2}$ for a contradiction.

$\square_{\text {Claim } 3}$

Claim 4 Let $x y$ be an edge in $H$. Then $e\left(\{x, y\}, T_{i}\right) \leq 2$ for $i=1,2$.

Proof of Claim 4: Suppose there exist three edges $E$ from $\{x, y\}$ to $T=T_{i}$ for some $i$. Let $u$ and $v$ be the hub vertices of $T$ and let $Q_{1}, Q_{2}, Q_{3}$ be the three paths of $T$, each including $u$ and $v$.

Suppose $u$ and $v$ are each incident to at least one edge of $E$ and assume the third edge is incident to a vertex $a_{1} \in Q_{1}$. Let $a=\operatorname{dist}_{Q_{1}}^{\prime}\left(u, a_{1}\right), b=\operatorname{dist}_{Q_{1}}^{\prime}\left(a_{1}, v\right), c=\operatorname{dist}_{Q_{2}}^{\prime}(u, v)$ and $d=\operatorname{dist}_{Q_{3}}^{\prime}(u, v)$ (recall that $\operatorname{dist}_{P}^{\prime}(u, v)$ is the defined to be the number of vertices between $u$ and $v$ along $P$ ). Note that the following arguments work regardless which of $u$ or $v$ is adjacent to which of $x$ or $y$. See Figure 2 for one case.

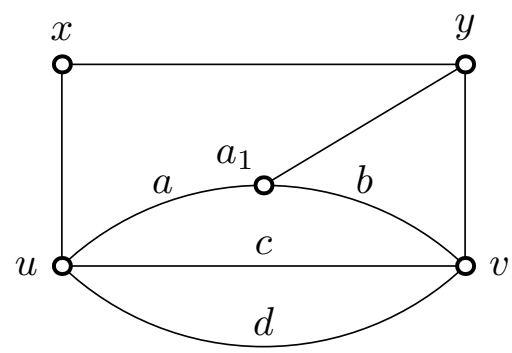

Figure 2: The structure of $T \cup\{x, y\}$. 
Notice that $a+b+1 \leq 2$ since otherwise $\left(T \backslash Q_{1}\right) \cup\{x, y, u, v\}$ would form a smaller $\theta$-graph. Similarly, $c+d \leq 2$ since $Q_{1} \cup\{x, y\}$ also forms a $\theta$-graph. This means that

$$
|T|=a+b+c+d+3 \leq 6
$$

which is a contradiction. Hence, both vertices $u$ and $v$ cannot be incident to edges of $E$. Since $a$ or $b$ may equal -1 , this argument also covers the case when $a_{1} \in\{u, v\}$.

Suppose only one of $u$ or $v$ (without loss of generality, suppose $u$ ) is incident to an edge of $E$. If both other edges of $E$ are incident to vertices $a_{1}$ and $a_{2}$ (in this order) on a single path (suppose $Q_{1}$ ) from $u$ to $v$, then we let $a=\operatorname{dist}_{Q_{1}}^{\prime}\left(u, a_{1}\right), b=\operatorname{dist}_{Q_{1}}^{\prime}\left(a_{1}, a_{2}\right)$, $c=\operatorname{dist}_{Q_{1}}^{\prime}\left(a_{2}, v\right), d=\operatorname{dist}_{Q_{2}}^{\prime}(u, v)$ and $e=\operatorname{dist}_{Q_{3}}^{\prime}(u, v)$. As above, $a+b+1 \leq 2$ since otherwise $T \cup\{x, y\}$ without the vertices between $u$ and $a_{2}$ forms a smaller $\theta$-graph. Similarly $c+d+e+1 \leq 2$. This implies that

$$
|T|=a+b+c+d+e+4 \leq 6
$$

which is a contradiction. If the other edges of $E$ are incident to vertices $a_{1} \in Q_{1}$ and $a_{2} \in$ $Q_{2}$, then we let $a=\operatorname{dist}_{Q_{1}}^{\prime}\left(u, a_{1}\right), b=\operatorname{dist}_{Q_{1}}^{\prime}\left(a_{1}, v\right), c=\operatorname{dist}_{Q_{2}}^{\prime}\left(u, a_{2}\right), d=\operatorname{dist}_{Q_{2}}^{\prime}\left(a_{2}, v\right)$ and $e=\operatorname{dist}_{Q_{3}}^{\prime}(u, v)$. Again we may easily see that $a+c \leq 2$ and $b+d+e+1 \leq 2$. This means that

$$
|T|=a+b+c+d+e+4 \leq 7
$$

which is again a contradiction. Hence, we may assume that no edges of $E$ are incident to hub vertices of $T$.

Now suppose all edges of $E$ are incident to vertices $a_{1}, a_{2}$ and $a_{3}$ (in order on a path from $u$ to $v$ ) in the interior of a single path (without loss of generality, suppose $Q_{1}$ ). If we let $a=\operatorname{dist}_{Q_{1}}^{\prime}\left(u, a_{1}\right), b=\operatorname{dist}_{Q_{1}}^{\prime}\left(a_{1}, a_{2}\right), c=\operatorname{dist}_{Q_{1}}^{\prime}\left(a_{2}, a_{3}\right), d=\operatorname{dist}_{Q_{1}}^{\prime}\left(a_{3}, v\right)$, $e=\operatorname{dist}_{Q_{2}}^{\prime}(u, v)$ and $f=\operatorname{dist}_{Q_{3}}^{\prime}(u, v)$, then we easily see that $a+d+e+f+2 \leq 2$. Conversely, $a, d, e, f \geq 0$ and one of $e$ or $f$ is at least 1 , which is a contradiction.

Next suppose two edges of $E$ are incident to vertices $a_{1}$ and $a_{2}$ (in order from $u$ to $v$ ) on a single path $Q_{1}$ and the third edge is incident to a vertex $a_{3}$ in $Q_{2}$. Let $a=\operatorname{dist}_{Q_{1}}^{\prime}\left(u, a_{1}\right), b=\operatorname{dist}_{Q_{1}}^{\prime}\left(a_{1}, a_{2}\right), c=\operatorname{dist}_{Q_{1}}^{\prime}\left(a_{2}, v\right), d=\operatorname{dist}_{Q_{2}}^{\prime}\left(u, a_{3}\right), e=\operatorname{dist}_{Q_{2}}^{\prime}\left(a_{3}, v\right)$ and $f=\operatorname{dist}_{Q_{3}}^{\prime}(u, v)$. By the same logic as before, we find:

$$
\left.\begin{array}{l}
a+d+f+1 \\
c+e+f+1 \\
a+b+1 \\
b+c+1 \\
d+e+1
\end{array}\right\} \leq 2
$$

meaning that $2(a+b+c+d+e+f) \leq 5$. This implies that

$$
|T|=a+b+c+d+e+f+5 \leq \frac{5}{2}+5<8
$$

which is a contradiction. 
Finally, suppose each of $a_{i}$ is on a different path $Q_{i}$ for each $i=1,2,3$. Let $a=$ $\operatorname{dist}_{Q_{1}}^{\prime}\left(u, a_{1}\right), b=\operatorname{dist}_{Q_{1}}^{\prime}\left(a_{1}, v\right), c=\operatorname{dist}_{Q_{2}}^{\prime}\left(u, a_{2}\right), d=\operatorname{dist}_{Q_{2}}^{\prime}\left(a_{2}, v\right), e=\operatorname{dist}_{Q_{3}}^{\prime}\left(u, a_{3}\right)$, $f=\operatorname{dist}_{Q_{3}}^{\prime}\left(a_{3}, v\right)$. Clearly $a+c+e+1 \leq 2$ and $b+d+f+1 \leq 2$ so

$$
|T|=a+b+c+d+e+f+5 \leq 7
$$

which is a contradiction, completing the proof of the claim.

Claim 5 Let $P=x y z$ be a path in $H$. Then $e\left(P, T_{i}\right) \leq 3$ holds for $i=1,2$.

Proof of Claim 5: $\quad$ Let $P=x y z$ be a path in $H$ and let $T=T_{i}$ be a $\theta$-graph such that $e(P, T) \geq 4$. First note that, by Claim 4 , we know $d_{T}(x)=d_{T}(z)=2$. Let $P_{1}, P_{2}$ and $P_{3}$ be the interiors of the paths from $u$ to $v$ in $T$ such that $\left|P_{1}\right| \leq\left|P_{2}\right| \leq\left|P_{3}\right|$.

We would first like to show that no vertex of $P$ can be adjacent to $u$ or $v$. Suppose $x$ is adjacent to $u$ and another vertex $w \in T$. Let $P^{\prime}$ be the path from $u$ to $w$ in $T$ (ends included). If $\left|P^{\prime}\right|>3$, then we could replace $P^{\prime}$ with uxw thereby creating a smaller $\theta$-graph. Similarly, if $\left|P_{2}\right| \geq 2$, we could remove $P_{2}$ from $T$ and add $x$, again creating a smaller $\theta$-graph. Hence, $\left|P_{1}\right| \leq\left|P_{2}\right| \leq 1$ and $\left|P^{\prime}\right| \leq 3$. This implies that $\left|P_{3}\right| \geq 4$ and $w \in P_{3}$.

If $z w^{\prime}$ is an edge for any vertex $w^{\prime}$ on the path $P^{\prime}$, then $P \cup P^{\prime}$ forms a $\theta$-graph of order 6 , a contradiction. If $z$ is adjacent to a vertex outside $P_{3} \cup u$, then $P \cup\left(T \backslash P_{3}\right)$ forms a $\theta$-graph of order at most 7 . This means that both adjacencies of $z$ must lie in $P_{3}$. Since $w \in P_{3}$, we know that $\left|P^{\prime}\right|=2$ and $\left|P_{1}\right|=0$ since otherwise $P_{3} \cup P$ forms a $\theta$-graph of order at most $|T|-1$.

This means that $\left|P_{3}\right| \geq 5$ and $z$ must be adjacent to the last two vertices in $P_{3}$ (directed from $u$ to $v$ ). Let $v^{-}$be the last vertex on $P_{3}$. Since we have shown $z v^{-}$is an edge, the graph induced on $P \cup P_{1} \cup P_{2} \cup\left\{u, v, v^{-}\right\}$forms a $\theta$-graph of order 7 . This is a contradiction, which implies that no vertex of $P$ is adjacent to $u$ or $v$, meaning all neighbors of $P$ on $T$ are in the interior of the paths of $T$.

If the neighborhood was smaller than 3 (meaning that only two vertices $w, w^{\prime} \in T$ ) are incident to all 4 edges coming from $P$, then $P \cup\left\{w, w^{\prime}\right\}$ forms a $\theta$-graph of order 5 . Hence, Fact 4 follows.

Fact $4\left|N_{T}(P)\right| \geq 3$.

First suppose that at least three of the edges from $P$ to $T$ fall into one path $P_{i}$. Clearly this path must be $P_{3}$ and this forces $\left|P_{1}\right|=0$ and $\left|P_{2}\right|=1$ since otherwise $P \cup P_{3}$ contains a $\theta$-graph of order at most $|T|-1$, a contradiction. This also implies that $\left|P_{3}\right| \geq 5$.

Let $u^{\prime}$ and $v^{\prime}$ be the vertices of $P_{3}$ which are adjacent to a vertex of $P$ and are closest to $u$ and $v$ respectively along $P_{3}$. The vertices $u^{\prime}$ and $v^{\prime}$ must be the ends of $P_{3}$ since otherwise $P$ and the subpath of $P_{3}$ from $u^{\prime}$ to $v^{\prime}$ would form a $\theta$-graph of order at most $|T|-1$. Let $P_{3}^{\prime}$ be the subpath of $P_{3}$ strictly between $u^{\prime}$ and $v^{\prime}$. Note that $\left|P_{3}^{\prime}\right| \geq 3$ and 
$x$ (or similarly $z$ ) cannot be adjacent to both $u^{\prime}$ and $v^{\prime}$ as this would allow us to replace the path $P_{3}^{\prime}$ with $x$, creating a $\theta$-graph of order at most $|T|-2$.

Without loss of generality, suppose $x u^{\prime}$ and $z v^{\prime}$ are edges. If all adjacencies of $P$ are in $P_{3}$, then $\left(P_{3}^{\prime} \backslash\left\{u^{\prime}\right\}\right) \cup P$ forms a $\theta$-graph of order at most $|T|-1$, a contradiction. Hence, suppose $x$ is adjacent to the single vertex of $P_{2}$. Then $P_{1} \cup P_{2} \cup\left\{x, u, u^{\prime}, v\right\}$ forms a $\theta$-graph or order 5 which is clearly a contradiction. Therefore, there is no path $P_{i}$ with 3 edges to $P$.

Now suppose two paths of $T$ have two edges each from $P$. If $e\left(x, P_{i}\right)=2$ and $e\left(z, P_{j}\right)=$ 2 , then the graph induced on $\left(T \backslash P_{j}\right) \cup\{x\}$ forms a $\theta$-graph of order at most $|T|-1$. Hence, we may suppose each of $x$ and $z$ have an edge to each of $P_{i}$ and $P_{j}$ for some $i, j \in\{1,2,3\}$. In following the proof of Claim 4, we consider the lengths of segments between these adjacencies.

Let $a$ be the number of vertices in the path $P_{k}$ for $k \notin\{i, j\}$, let $b$ be number of vertices between $u$ and the first adjacency of $P$ on $P_{i}$, let $c$ be the number of vertices between the adjacencies on $P_{i}$ and let $d$ be the number of vertices between the second adjacency on $P_{i}$ and $v$. Similarly define $e, f$ and $g$ for the numbers of vertices on $P_{j}$ and see Figure 3 . Let $w_{1}, w_{2}, w_{3}, w_{4}$ be as in Figure 3. The following proof works regardless of which adjacency $(x$ or $z$ ) comes first in these paths so we assume the case pictured in Figure 3.

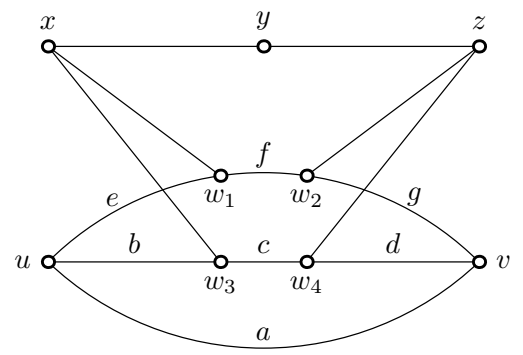

Figure 3: The structure of $T \cup P$.

First note that $b+c \leq 0, c+d \leq 0, e+f \leq 0$ and $f+g \leq 0$ since we could remove the segment between $u$ and $w_{2}$ and replace a single vertex $x$ or $z$. By assumption, $b, d, e, g \geq 0$. Also, by Fact 4 , at least one of $c$ or $f$ is at least 0 so suppose $c=0$ which implies $b=d=0$. Since $P_{k} \cup P_{i} \cup P$ forms a $\theta$-graph, $a \geq 1$. Conversely, $a+b+d+e+g \leq 1$ since the segments $w_{1} \ldots w_{2}$ and $w_{3} \ldots w_{4}$ along with $P$ form a $\theta$-graph. This, along with the fact that $b, d, e, g \geq 0$, implies that $a \leq 1$. Hence, $a=1$.

Recall that $e+f \leq 0$ and $f+g \leq 0$ and $f, g \geq 0$. This means that $f \leq 0$. Then since the segments $w_{1} \ldots w_{2}$ and $w_{3} \ldots w_{4}$ along with $P$ form a $\theta$-graph, $f \geq 1$ which is a contradiction.

Finally we consider the case where two edges from $P$ fall into one path $P_{i}$ and one to each of the other paths. If $P_{i}$ receives two edges from a single vertex ( $z \cup\left(T \backslash P_{i}\right)$ forms a $\theta$-graph on at most $|T|-1$ vertices, a contradiction. This means that $P_{i}$ receives an edge from each of $x$ and $z$. If we remove $P_{j}$ for some $j \neq i$, what remains contains a $\theta$-graph using only one of $x$ or $z$. This means that $\left|P_{j}\right|=1$ for each $j \neq i$. 
Conversely, if we remove $P_{i}$, what remains is also a $\theta$-graph. This implies that $\left|P_{i}\right| \leq 3$. Since $T=P_{1} \cup P_{2} \cup P_{3} \cup\{u, v\}$, we find that $|T| \leq 7$ which is a contradiction, completing the proof of Claim 5 .

Claim 5

Claim 6 If $P=x y z$ is a path in $H$ with $x z \notin E(G)$ and $\{x, z\} \subseteq V_{4}$, then $e\left(P, T_{i}\right) \leq 2$ holds for $i=1,2$.

Proof of Claim 6: The proof of this claim follows similarly to the proof of Claim 4. Suppose there are three edges $E$ from $P$ to $T=T_{i}$ for some $i$. Let $u$ and $v$ be the hub vertices of $T$ and let $Q_{1}, Q_{2}$ and $Q_{3}$ be the paths of $T$ each containing $u$ and $v$.

Suppose $u$ and $v$ are each incident to at least one edge of $E$ and, without loss of generality, that the third edge of $E$ is incident to a vertex $a_{1} \in Q_{1}$. Again define $a, b, c, d$ similarly to Figure 2. In this situation $a+b+1 \leq 3$ since we could replace the interior of $Q_{1}$ with $P$. Conversely, $P \cup Q_{1}$ is a $\theta$-graph of order at most $8<10$ containing two non adjacent vertices of $V_{4}$. This contradicts Claim 3. Hence, $u$ and $v$ cannot both be incident to edges of $E$.

Now suppose one of $u$ or $v$ (without loss of generality $u$ ) is incident to an edge of $E$. If both other edges of $E$ are incident to vertices $a_{1}, a_{2}$ in a single path (suppose $Q_{1}$ and suppose $a_{1}$ appears before $a_{2}$ on the path from $u$ to $v$ ), then let $a=\operatorname{dist}_{Q_{1}}^{\prime}\left(a_{1}, a_{2}\right)$ and $b=\operatorname{dist}_{Q_{1}}^{\prime}\left(a_{2}, v\right)$. If we let $Q_{1}^{\prime}$ be the segment strictly between $a_{1}$ and $v$ on $Q_{1}$, we see immediately that $a+b \geq 4$ since $P \cup Q_{1}^{\prime} \cup\left\{a_{1}, v\right\}$ forms a $\theta$-graph and this must have order at least 10. Conversely then $a+b+1 \leq 3$ since $P \cup\left(T \backslash Q_{1}^{\prime}\right)$ forms a $\theta$-graph and this must also have order at least 10 (by Claim 3). This is clearly a contradiction.

Hence, suppose $u$ is still incident to an edge of $E$ but the other vertices $a_{i}$ incident to edges of $E$ are on paths $Q_{i}$ respectively for $i=1,2$. Let $a=\operatorname{dist}_{Q_{1}}^{\prime}\left(a_{1}, v\right)$ and let $b=\operatorname{dist}_{Q_{2}}^{\prime}\left(a_{2}, v\right)$. Just as in the previous case, $a+b \leq 3$ but conversely, $a+b \geq 4$ which is again a contradiction.

This means we may suppose that neither $u$ nor $v$ is incident to any edges of $E$. Let $a_{1}, a_{2}$ and $a_{3}$ be the vertices of $T$ incident to edges of $E$ and first suppose $a_{i} \in Q_{1}$ for all $i$ (and suppose they appear in order along $Q_{1}$ ). If we let $a=\operatorname{dist}_{Q_{1}}^{\prime}\left(a_{1}, a_{3}\right)$, then as in the previous cases, we easily get $a \leq 3$ but also $a \geq 5$ which is another contradiction.

Next suppose two edges of $E$ are incident to $a_{1}, a_{2} \in Q_{1}$ and the third edge of $E$ is incident to $a_{3} \in Q_{2}$. Let $a=\operatorname{dist}_{Q_{1}}^{\prime}\left(u, a_{1}\right), b=\operatorname{dist}_{Q_{1}}^{\prime}\left(a_{1}, a_{2}\right), c=\operatorname{dist}_{Q_{1}}^{\prime}\left(a_{2}, v\right)$, $d=\operatorname{dist}_{Q_{2}}^{\prime}\left(u, a_{3}\right), e=\operatorname{dist}_{Q_{2}}^{\prime}\left(a_{3}, v\right)$ and $f=\operatorname{dist}_{Q_{3}}^{\prime}(u, v)$. Again the following argument works regardless which of $x, y$ or $z$ is adjacent to which $a_{i}$. See Figure 4.

In this case, by the same arguments, we find:

$$
\left.\begin{array}{r}
a+b+1 \\
b+c+1 \\
a+d+f+1 \\
c+e+f+1 \\
d+e+1
\end{array}\right\} \leq 3
$$




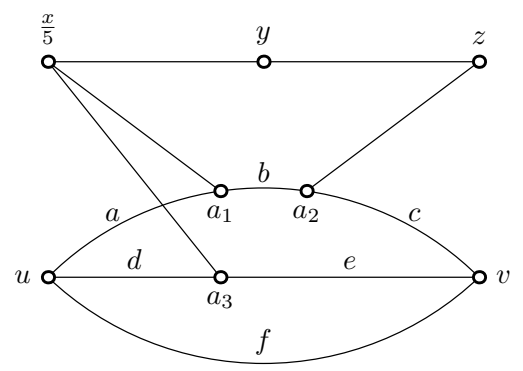

Figure 4: The structure of $T \cup P$.

and regardless which of $x, y$ or $z$ is adjacent to which of $a_{1}, a_{2}$ or $a_{3}$, at least one of these inequalities must be strict. This implies that $|T|=a+b+c+d+e+f+5<10$ which is again a contradiction to Claim 3.

Finally, suppose each of $a_{i}$ is in $Q_{i}$ for $i=1,2,3$. Let $a=\operatorname{dist}_{Q_{1}}^{\prime}\left(u, a_{1}\right), b=$ $\operatorname{dist}_{Q_{1}}^{\prime}\left(a_{1}, v\right), c=\operatorname{dist}_{Q_{2}}^{\prime}\left(u, a_{2}\right), d=\operatorname{dist}_{Q_{2}}^{\prime}\left(a_{2}, v\right), e=\operatorname{dist}_{Q_{3}}^{\prime}\left(u, a_{3}\right)$ and $f=\operatorname{dist}_{Q_{3}}^{\prime}\left(a_{3}, v\right)$. Again we see that $a+c+e+1 \leq 3$ and $b+d+f+1 \leq 3$ which means that $|T|=$ $a+b+c+d+e+f+5 \leq 9$. This is a contradiction, completing the proof of the claim.

$\square_{\text {Claim } 6}$

Claim 7 Let $S=x y z$ be a triangle in $H$ such that $\{y, z\} \cap V_{4} \neq \emptyset$ and $d_{H}(y)=d_{H}(z)=2$. Then $y, z \in V_{4}$ and $e\left(y, T_{1}\right)=e\left(z, T_{2}\right)=2$ (i.e. $y$ and $z$ are in $\left.V_{4}\right)$.

Proof of Claim 7: $\quad$ Since we assume $y$ and $z$ each have at least 2 edges to $T_{1} \cup T_{2}$, suppose $y$ and $z$ each have at least one edge to $T_{i}$ (without loss of generality, suppose $i=1$ ). Let $u$ and $v$ be vertices of $T_{1}$ adjacent to $y$ and $z$ respectively. Clearly $u \neq v$ and, in order to avoid a $\theta$-graph of order less than 8 , the distance between $u$ and $v$ is at least 4 . The diameter of $T_{1}$ is at most $\frac{\left|T_{1}\right|}{2}$ so if $\left|T_{1}\right|>8$, we may replace $T_{1}$ with a smaller $\theta$-graph using the triangle $x y z$ and a shortest path through $T_{1}$ from $u$ to $v$, a contradiction.

Hence, suppose $\left|T_{1}\right|=8$. We may still replace $T_{1}$ as above with another $\theta$-graph of order 8 using the triangle $x y z$. Since $\{y, z\} \cap V_{4} \neq \emptyset$, this contradicts Claim 3 part $(i)$. This means that $y$ and $z$ cannot each have an edge to a single $\theta$-graph $T_{i}$.

If either $y$ or $z$ (suppose $Y$ ) has at least 3 edges to $T_{1} \cup T_{2}$, then $y$ has 3 edges to a single $\theta$-graph which is a contradiction to Claim 4 .

$\square_{\text {Claim } 7}$

Claim 8 Let $S_{1}, S_{2}$ be two disjoint special triangles. Then $e\left(S_{1}, S_{2}\right)=0$.

Proof of Claim 8: Let $S_{i}=x_{i} y_{i} z_{i}$ with central vertex $x_{i}$ for $i=1,2$. By the definition of special triangles, there are no edges from $S_{i} \backslash x_{i}$ to $S_{j}$ for $i \neq j$. Hence, suppose the edge $x_{1} x_{2} \in E(G)$. By Claim 7, we get, without loss of generality, $e\left(y_{1}, T_{1}\right)=$ $e\left(z_{1}, T_{2}\right)=e\left(y_{2}, T_{1}\right)=e\left(z_{2}, T_{2}\right)=2$. Let $\left\{v_{i}, v_{i}^{\prime}\right\}=N\left(y_{i}\right) \cap T_{1}$ for $i=1,2$. 
Let $C_{1}$ be the smallest cycle of $T_{1}$ containing both $v_{1}$ and $v_{1}^{\prime}$. Since $C_{1} \cup y_{1}$ forms a $\theta$-graph containing a vertex $\left(y_{1}\right)$ of $V_{4}$, Claim 3 part $(i)$ implies that $\left|C_{1}\right| \geq 9$. Certainly the same holds for a similarly defined cycle $C_{2}$. If $\left|C_{1}\right|=9$ and $v_{2}, v_{2}^{\prime} \in C_{1}$, then, without loss of generality, there exists a path $P$ from $v_{1}$ to $v_{2}$ containing at most 2 intermediate vertices. At this point, the vertices of $S_{1} \cup P \cup\left\{v_{1}, v_{2}, y_{2}, x_{2}\right\}$ form a $\theta$-graph of order 9 containing two nonadjacent vertices $\left(y_{1}\right.$ and $\left.y_{2}\right)$ of $V_{4}$, contradicting Claim 3 part (ii). This implies that $\left|T_{1}\right| \geq 10$. This argument also implies the following fact which will be used later.

Fact 5 The shortest distance between sets $\left\{v_{1}, v_{1}^{\prime}\right\}$ and $\left\{v_{2}, v_{2}^{\prime}\right\}$ on $T_{1}$ is at least 4 .

Consider two distinct pairs $A$ and $B$ of vertices (for example, see $\left\{v_{1}, v_{1}^{\prime}\right\}$ and $\left\{v_{2}, v_{2}^{\prime}\right\}$ ) in a $\theta$-graph $T$. The following fact follows immediately.

Fact 6 In a $\theta$-graph $T$, the shortest distance between a vertex $a \in A$ and a vertex $b \in B$ is at most $\frac{|T|-2}{2}$.

Suppose $\left|T_{1}\right|=10$ and let $A=\left\{v_{1}, v_{1}^{\prime}\right\}$ and $B=\left\{v_{2}, v_{2}^{\prime}\right\}$. By Facts 5 and 6 , there exist two paths of length exactly 4 one between, without loss of generality, the pair of vertices $v_{1}, v_{2}$ and the other between the pair $v_{1}^{\prime}, v_{2}^{\prime}$. This forces $T_{1}$ to be a chorded cycle and, to avoid shortening one of the aforementioned paths, the chord must go from one such path to the other. This implies $\left|C_{i}\right| \leq 8$ for some $i=1,2$, which is a contradiction. Hence, $\left|T_{1}\right| \geq 11$.

By Fact 6 , there exists a path $P$, without loss of generality between $v_{1}$ and $v_{2}$, with at most $\frac{|T|-4}{2}$ internal vertices. The vertices of $S_{1} \cup P \cup\left\{v_{1}, v_{2}, y_{2}, x_{2}\right\}$ again form a $\theta$-graph $T$. Since $\left|T_{1}\right| \geq 11$, we have:

$$
|T| \leq 7+|P| \leq 7+\frac{\left|T_{1}\right|-4}{2}<\left|T_{1}\right|
$$

which is a contradiction, completing the proof of this claim.

Claim 8

Fact 7 Let $S=x y z x$ be a special triangle with a central vertex $x$ in $H$. Then $e\left(y, T_{i}\right)=$ $e\left(z, T_{j}\right)=2$ for $\{i, j\}=\{1,2\}$.

This fact follows immediately from Claim 7 .

Claim 9 Let $S=x y z x$ be a special triangle with a central vertex $x$ in $H$. Then for any $v \in N_{G}(x) \cap H-\{y, z\}, d_{H}(v) \geq 3$.

Proof of Claim 9: $\quad$ By Fact 7, without loss of generality, the $y$ and $z$ each have two edges to $T_{1}$ and $T_{2}$ respectively. Let $v$ be as given in the statement. If $v$ has at most one edge to $T_{1} \cup T_{2}$, then $d_{H}(v) \geq 3$ so suppose $v$ has an edge to each of $T_{1}$ and $T_{2}$. By Claim 5 using the path $v x y$ or $v x z$, the vertex $v$ cannot have more than one edge to either 
of $T_{1}$ or $T_{2}$. If $v \in V_{4}$, then the path $v x y$ is a contradiction to Claim 6 since $v y \notin E(G)$. Hence $v \notin V_{4}$. That means that $d(v) \geq 5$ but we already observed that $d_{T_{1} \cup T_{2}}(v) \leq 2$ so $d_{H}(v) \geq 3$.

Claim 9

Let $P=x_{1} x_{2} \ldots x_{\ell}$ be a longest path in $H$. By the choice of $T_{1}$ and $T_{2}$, note that $\ell \geq 2$. When $d_{H}\left(x_{1}\right) \geq 2$, note that there exists a vertex $x_{t} \in P$ with $3 \leq t \leq \ell$ such that $x_{1} x_{t} \in E(G)$. We may assume that $P$ is chosen so that $d_{H}\left(x_{1}\right)$ is minimum, and subject to this condition, if we have $d_{H}\left(x_{2}\right)=2$, we prefer choosing $P$ so that $t$ is maximum. Since $H$ does not contain a $\theta$-graph, note that $d_{H}\left(x_{1}\right)=1$ or 2 . According to this, we divide our proof into two cases.

Case $1 d_{H}\left(x_{1}\right)=1$.

We begin this case with a helpful claim.

Claim $10 x_{1} \in V_{4}$.

Proof of Claim 10: Assume $x_{1} \notin V_{4}$. Then, since $e\left(x_{1}, T_{1} \cup T_{2}\right) \geq 4$, in view of Claim $4, e\left(x_{2}, T_{1} \cup T_{2}\right)=0$. Since $H$ does not contain a $\theta$-graph, we have $e\left(x_{2}, P\right) \leq 3$.

Suppose that $e\left(x_{2}, P\right)=3$. Then, it follows that $e\left(x_{2}, P \backslash\left\{x_{1}, x_{2}, x_{3}\right\}\right)=1$. Since $\delta(G) \geq 4$, there is a vertex $v \in H \backslash P$ such that $v x_{2} \in E(G)$. Since $d_{H}\left(x_{1}\right)=1, e\left(x_{2}, P \backslash\right.$ $\left.\left\{x_{1}, x_{2}, x_{3}\right\}\right)=1$ and $H$ does not contain a $\theta$-graph, and moreover, $P$ is a longest path in $H$, this implies $d_{H}(v)=1$, and hence $e\left(v, T_{1} \cup T_{2}\right) \geq 3$ holds. Let us consider the edges between the path $P^{\prime}=v x_{2} x_{1}$ and $T_{1} \cup T_{2}$. We see that $e\left(P^{\prime}, T_{1} \cup T_{2}\right) \geq 7$. However, this implies that we get a contradiction to Claim 5 for some $i \in\{1,2\}$.

Thus we may assume that $e\left(x_{2}, P\right)=2$. Then, by Claim 4 , there exist two vertices $v, v^{\prime} \in N_{G}\left(x_{2}\right) \cap(H \backslash P)$. Since $P$ is a longest path in $H$, note that $v v^{\prime} \notin E(G)$. Also, since $H$ does not contain a $\theta$-graph and all edges from $v$ and $v^{\prime}$ to $H$ must go to $P$ (since $P$ is longest), it is easy to see that $\min \left\{d_{H}(v), d_{H}\left(v^{\prime}\right)\right\}=1$. By symmetry, we may assume that $d_{H}(v)=1$. Consequently, arguing similarly as above by putting $P^{\prime}=v x_{2} x_{1}$, we get a contradiction to Claim 5 .

$\square$ Claim 10

Suppose that there exists a vertex $v$ such that $v \notin P$ and $v x_{2} \in E(G)$ since $P$ is a longest path, we have $v x_{1} \notin E(G)$. By the symmetry of the roles of $x_{1}$ and $v$ (note that $P^{\prime}=v x_{2} x_{3} \ldots x_{\ell}$ could be another longest path in $H$ ), in view of Claim 10, we may assume that $v \in V_{4}$. Since $H$ does not contain a $\theta$-graph, we see that $e\left(v, T_{1} \cup T_{2}\right) \geq 2$. Then, by considering a path $P^{*}=x_{1} x_{2} v$, we get a contradiction to Claim 5 . Hence, we may assume that there is no such vertex $v$. Since $H$ does not contain a $\theta$-graph and $\delta(G) \geq 4$, in view of Claim 4 , we obtain $x_{2} \in V_{4}, d_{P}\left(x_{2}\right)=3$ and $e\left(x_{2}, T_{1} \cup T_{2}\right)=1$. By symmetry, we may assume that $e\left(x_{2}, T_{1}\right)=1$.

Claim $11 d_{p}\left(x_{3}\right)=2$. 
Proof of Claim 11: Note that $d_{H}\left(x_{1}\right)=1$ and $d_{P}\left(x_{2}\right)=3$. Since $H$ does not contain a $\theta$-graph, the assertion follows easily.

$\square_{\text {Claim } 11}$

Claim 12 In $H$, there is no triangle $S$ which contains $x_{3}$.

Proof of Claim 12: $\quad$ First suppose that there is a special triangle $S$ with central vertex $x_{3}$ in $H$. Then, in view of Fact 7 , there is a vertex $p \in S$ such that $p \in V_{4}$ and $e\left(p, T_{1}\right)=2$. However, by considering a path $P^{*}=x_{2} x_{3} p$, this contradicts Claim 5 .

Hence, to prove this Claim, we have only to consider the case where $H$ has a triangle $S$ such that $x_{3} \in S$ and $S$ is not a special triangle. We see from Claim 11 that $S \cap P=\left\{x_{3}\right\}$. Also, since $P$ is a longest path in $H$, it follows that $d_{H}(v)=2$ for any $v \in S \backslash x_{3}$. However, since we know that $S$ is not a special triangle, this implies that $e\left(S-x_{3}, T_{1} \cup T_{2}\right) \geq 5$, which will lead us to a contradiction to Claim 4.

$\square_{\text {Claim } 12}$

In view of Claims 4-6 since $e\left(\left\{x_{1}, x_{2}\right\}, T_{1} \cup T_{2}\right)=4$, it is easy to check that $d_{H}\left(x_{3}\right) \geq 4$ by considering the cases where $x_{3} \in V_{4}$ and $x_{3} \notin V_{4}$ separately. Then, there exist two distinct vertices $u, v \in H \backslash P$ such that $u, v \in N_{G}\left(x_{3}\right)$. By Claim 12, we see that $u v \notin$ $E(G)$.

Suppose that there exists a vertex $w \in H-P$ such that $N_{G}(w) \cap\{u, v\} \neq \emptyset$, say, $w v \in E(G)$. Since $P$ is a longest path in $H$ and $H$ does not contain a $\theta$-graph, this together with Claim 12 implies that $d_{H}(w)=1$. By the symmetry of the roles of $P$ and a path $P^{*}=w v x_{3} x_{4} \ldots x_{\ell}$, we see that $d_{P^{*}}(v)=3$ (note that $v$ and $x_{2}$ play the same role). However, then we can find a $\theta$-graph in $H$, a contradiction. Thus we may assume that there is no such a vertex like $w$.

Since we had $u v \notin E(G)$, this means that $e\left(u, T_{1} \cup T_{2}\right) \geq 3$ and $e\left(v, T_{1} \cup T_{2}\right) \geq 3$. Then, considering a path $P^{*}=u x_{3} v$, we can easily get a contradiction to Claims 5 and 6 . This completes the proof of Case 1.

Case $2 d_{H}\left(x_{1}\right)=2$.

In this case, let $x_{t}$ be a vertex in $P$ such that $x_{1} x_{t} \in E(G)$. Since $H$ does not contain a $\theta$-graph, we see from Claim 4 and $x_{1} x_{t} \in E(G)$ that $x_{1}, x_{2} \in V_{4}$ and $d_{H}\left(x_{2}\right)=2$.

Fact 8 For any $1 \leq i \leq t-1$, we have $d_{P}\left(x_{i}\right)=2$.

Here we further divide the proof into two subcases.

Subcase $2.1 t \geq 4$.

Since $P$ is a longest path in $H$ and $H$ has no $\theta$-graph, we see that $d_{H}\left(x_{t-1}\right)=2$ and $e\left(x_{t-1}, T_{1} \cup T_{2}\right) \geq 2$. Suppose that $x_{t-1} \notin V_{4}$ so $e\left(x_{t-1}, T_{1} \cup T_{2}\right) \geq 3$. Then, in view of Claim 5 and Fact 8, it follows that $t \geq 5$. Since $d_{P}\left(x_{t-2}\right)=2$ (to avoid a $\theta$ graph), in view of Claim 4 , we have $e\left(x_{t-2}, T_{1} \cup T_{2}\right) \leq 1$. Hence, there exists a vertex 
$u \in H \backslash P$ such that $u x_{t-2} \in E(G)$. Since $H$ does not contain a $\theta$-graph and $P$ is a longest path in $H$, it follows that $d_{H}(u)=1$. However, then by replacing $P$ by another path $P^{*}=u x_{t-2} x_{t-3} \ldots x_{1} x_{t} x_{t+1} \ldots x_{\ell}$, we get a contradiction to the choice of $P$ (in this case).

Thus we have $x_{t-1} \in V_{4}$ and $e\left(x_{t-1}, T_{1} \cup T_{2}\right)=2$. Again, in view of Claim 5 , we have $t \geq 5$, and arguing similarly as above, we see that $x_{t-2} \in V_{4}$ and $e\left(x_{t-2}, T_{1} \cup T_{2}\right)=2$. Then, we can repeat using Claim 5 and we get $t \geq 6$. Recall that $d_{P}\left(x_{t-3}\right)=2$.

Suppose that there exist two distinct vertices $u, w \in H \backslash P$ such that $u w, w x_{t-3} \in E(G)$. By the choice of $P$, it is easy to see that $d_{H}(u)=d_{H}(w)=2$ since $u$ must have an edge to $P$ by the case we're in, and this edge must go to $x_{t-3}$ to avoid a $\theta$-graph. Since $H$ does not contain a $\theta$-graph and $P$ is a longest path in $H$, we see that $u x_{t-3} \in E(G)$. Also, in view of Claim 4 , we get $u, w \in V_{4}$. Hence, $\left\langle u, w, x_{t-3}\right\rangle$ forms a special triangle with a central vertex $x_{t-3}$. However, this contradicts Claim 9 because $d_{H}\left(x_{t-2}\right)=2$.

In view of the above observation, we must have one of the following cases:

(i) There exist two vertices $u, w \in H \backslash P$ such that $x_{t-3} u, x_{t-3} w \in E(G)$ and $u w \notin E(G)$.

(ii) $x_{t-3} \in V_{4}$ and $e\left(x_{t-3}, T_{1} \cup T_{2}\right) \geq 1$.

If (i) happens, then we must have $e\left(u, T_{1} \cup T_{2}\right) \geq 3$ and $e\left(w, T_{1} \cup T_{2}\right) \geq 3$ because $d_{H}(u)=d_{H}(w)=1$. However, then by considering a path $P^{*}=w x_{t-3} u$, we get a contradiction to Claim 5 (note that when $e\left(u, T_{1} \cup T_{2}\right)=e\left(w, T_{1} \cup T_{2}\right)=3$, it follows that $\left.u, w \in V_{4}\right)$. Also, if (ii) happens, let $u$ be a vertex in $H \backslash P$ such that $u x_{t-3} \in E(G)$. Then, by considering a path $P^{*}=x_{t-3} x_{t-2} x_{t-1}$, we get a contradiction to Claim 5 . This completes the proof of the subcase.

Subcase $2.2 t=3$.

Using the fact that $P$ is a longest path in $H$ and Claim 4, it is easy to check that $\left\langle x_{1}, x_{2}, x_{3}\right\rangle$ forms a special triangle with a central vertex $x_{3}$. Suppose that there exists a vertex $u \in H \backslash P$ such that $u x_{3} \in E(G)$. Then by the choice of $P$, note that $\ell \geq 4$. Applying Claim 9 to the special triangle $\left\langle x_{1}, x_{2}, x_{3}\right\rangle$ and $u \in N_{G}\left(x_{3}\right)$, we see that there is a vertex $w \in H \backslash P$ such that $u w \in E(G)$. Then, by the symmetry of the roles of $P$ and a path $P^{*}=w u x_{3} x_{4} \ldots x_{\ell}$, we have $\left\langle w, u, x_{3}\right\rangle$ forms a special triangle with a central vertex $x_{3}$. However, this contradicts Claim 9.

Thus we have $d_{H}\left(x_{3}\right)=d_{P}\left(x_{3}\right)$. This together with Claim 4 and Fact 7 implies $x_{3} \in V_{4}$ and $d_{P}\left(x_{3}\right)=d_{G}\left(x_{3}\right)=4$. Hence, there exists a vertex $x_{s} \in P$ such that $x_{3} x_{s} \in E(G)$ and $4<s \leq \ell$.

Suppose that there exists another longest path $P^{*}=x_{1}^{\prime} x_{2}^{\prime} x_{3}^{\prime} x_{4} x_{5} \ldots x_{\ell}$ such that $\left\{x_{1}^{\prime}, x_{2}^{\prime}, x_{3}^{\prime}\right\} \cap P=\emptyset$. Then, by the symmetry of the roles of $x_{i}$ and $x_{i}^{\prime}$ for $i=1,2,3$, it follows that $\left\langle\left\{x_{1}^{\prime}, x_{2}^{\prime}, x_{3}^{\prime}\right\}\right\rangle$ forms a special triangle in $H$ and $d_{H}\left(x_{3}^{\prime}\right)=d_{P}\left(x_{3}^{\prime}\right)$. This forces $e\left(x_{3}^{\prime}, P \backslash\left\{x_{1}, x_{2}, x_{3}\right\}\right)=2$. Then, $\left\langle P \cup\left\{x_{3}^{\prime}\right\}\right\rangle$ contains a $\theta$-graph, a contradiction. Hence we may assume that there is no such a path like $P^{*}$.

Next suppose there exists a path $P^{*}=x_{2}^{\prime} x_{3}^{\prime} x_{4} x_{5} \ldots x_{\ell}$ such that $\left\{x_{2}^{\prime}, x_{3}^{\prime}\right\} \cap P=\emptyset$. Suppose that $x_{2}^{\prime} x_{4} \in E(G)$. Then, by the above observation about another longest path 
and in view of Claim 4 , it is easy to check that $\left\langle\left\{x_{2}^{\prime}, x_{3}^{\prime}, x_{4}\right\}\right\rangle$ forms a special triangle. However, this contradicts Claim 8. So we have $x_{2}^{\prime} x_{4} \notin E(G)$.

Since $H$ does not contain a $\theta$-graph and $x_{3} x_{s} \in E(G)$, we can easily see that $d_{H}\left(x_{2}^{\prime}\right)=$ 1 , and hence $e\left(x_{2}^{\prime}, T_{1} \cup T_{2}\right) \geq 3$. In view of Claim 4 , this implies $d_{H}\left(x_{3}^{\prime}\right) \geq 3$ and also we see that $e\left(x_{3}^{\prime}, P\right)=1$ because $H$ does not contain a $\theta$-graph. This means that there exists a path $P^{\prime \prime}=x_{2}^{\prime} x_{3}^{\prime} v$ such that $v \notin P$ and $e\left(P^{\prime \prime}, T_{1} \cup T_{2}\right) \geq 6$ and the equality holds only if $x_{2}^{\prime}, v \in V_{4}$. This will lead us to a contradiction to Claim 6 .

Since $x_{3} x_{s} \in E(G)$ and $H$ does not contain a $\theta$-graph, we see that $d_{P}\left(x_{4}\right)=2$. Consequently, if $x_{4} \in V_{4}$, this together with Claim 6 and the above observation about a path distinct from $P$ shows that $d_{T_{1} \cup T_{2}}\left(x_{4}\right)=0$ and there are two distict vertices $u, v \in H \backslash P$ such that $u v \notin E(G), u, v \in N_{G}\left(x_{4}\right)$ and $d_{H}(u)=d_{H}(v)=1$. Then by considering a path $P^{*}=u x_{4} v$, we get a contradiction to Claim 6 because $e\left(P^{*}, T_{1} \cup T_{2}\right) \geq 6$ and the equality holds only if $u, v \in V_{4}$.

If $x_{4} \notin V_{4}$, by Claim 5 and Fact 7 , it is easy to check that $e\left(x_{4}, T_{i}\right) \leq 1$ for each $i$. If $e\left(x_{4}, T_{i}\right)=0$ for some $i$, then there exist two vertices $u$ and $v$ as in the previous paragraph. Hence, suppose $e\left(x_{4}, T_{i}\right)=1$ for each $i$. Since $x_{4} \notin V_{4}$, there exists a vertex $u$ in $N\left(x_{4}\right) \cap(H \backslash P)$. Again $d_{H}(u)=1$ so $e\left(u, T_{1} \cup T_{2}\right) \geq 3$. Since $e\left(x_{4}, T_{i}\right)=1$ for each $i$, we get $e\left(\left\{x_{4}, u\right\}, T_{1} \cup T_{2}\right) \geq 5$ which contradicts Claim 4 . This completes the proof of the subcase and the proof of our main result.

\section{Further Results}

We now prove a more general, although much weaker upper bound.

Theorem 15 Given integers $k \geq 3$ and $\alpha \geq 6$, we get $f(k, \alpha) \leq 2 k \alpha-10$.

Proof: Suppose $G$ is a graph of order $n=2 k \alpha-9$ with $\alpha(G) \leq \alpha$ containing no $k$ disjoint $\theta$-graphs.

Claim 13 The order of the smallest $\theta$-graph in $G$ is at least 13.

Proof of Claim 13: Suppose there exists a $\theta$-graph $T$ in $G$ with $|T| \leq 12$. Let $H=G \backslash T$. If $k \geq 4$, then $|H|=|G|-|T| \geq|G|-12=2 k \alpha-9-12 \geq 2(k-1) \alpha-9$ since $\alpha \geq 6$ and we may apply induction. The base case of this induction is when $k=3$ and $|G|=2 k \alpha-9=6 \alpha-9>3 \alpha+8=f(3, \alpha)$.

$\square_{\text {Claim } 13}$

Claim 14 The graph $G$ has minimum degree $\delta(G) \geq 2 k+1$.

Proof of Claim 14: Let $v$ be a vertex with $d(v) \leq 2 k$ and let $H$ be the graph obtained from $G$ by contracting $v \cup N(v)$ to a new vertex $w$. If $w$ is not in a maximum independent set of $H$, then this independent set can be extended to an independent set of $G$ containing the vertex $v$. There exist two independent vertices in $N(v)$ because $d(v) \geq 4$ and $G$ contains no $K_{4}^{-}$(by induction on $k$ ). Any maximum independent set of 
$H$ containing $w$ corresponds to an independent set of $G$ containing at least 2 independent vertices of $N(v)$. Hence, $\alpha(H) \leq \alpha(G)-1$ and $|H| \geq|G|-2 k$ so we apply induction on $\alpha$ when $\alpha \geq 7$. If $\alpha=6,|G|=2 k \alpha-9=12 k-9>3 \alpha+4 k-4$ since $k \geq 4$ which is a contradiction.

Assume $G$ is an edge-maximal counterexample. This means $G$ contains a spanning subgraph which is the union of $k-1$ disjoint $\theta$-graphs $T_{1}, \ldots, T_{k-1}$ and a $\theta$-free graph $H$. Let $T=\cup_{i=1}^{k-1} T_{i}$. Choose the set of $\theta$-graphs in $G$ such that $|T|$ is minimum. We must first show that $|H| \geq 1$. This follows by induction on $k$ since $f(k-1, \alpha)+1 \leq$ $2(k-1) \alpha-9<2 k \alpha-9$ for $k \geq 5$. The base case of this induction is when $k=4$ and $f(k-1, \alpha)=3 \alpha+8<8 \alpha-9=2 k \alpha-9$ since $\alpha \geq 6$.

If $\delta(H) \geq 3$, there would exist a $\theta$-graph in $H$, so there must exist a vertex $v \in H$ with $d_{H}(v) \leq 2$. This implies that $d_{T}(v) \geq 2 k-1$ and $d_{T_{i}}(v) \geq 3$ for some $1 \leq i \leq k-1$. Since $\left|T_{i}\right| \geq 13$, there exists a $\theta$-graph $T_{i}^{\prime}$ in the graph induced on $T_{i} \cup v$ such that $\left|T_{i}^{\prime}\right|<\left|T_{i}\right|$ which contradicts the minimality of $|T|$.

Define $f(k, \alpha, \delta)$ to be the maximum order of a graph $G$ with $\alpha(G) \leq \alpha, \delta(G) \geq \delta$ and no $k$ disjoint $\theta$-graphs. Similar to Theorem 5 , we prove the following result concerning $f(k, \alpha, \delta)$. This result shows that, if we assume a reasonable minimum degree condition, the upper bound of Theorem 3 can be extended beyond the given restrictions on $k$ and $\alpha$.

Theorem 16 For all integers $k$ and $\alpha, f(k, \alpha, 3 k-1) \leq 3 \alpha+4 k-4$.

Proof: The result is trivial for $\alpha=1$ and it follows from Theorem 3 for $\alpha \leq 5$ and $k \leq 3$ so suppose $\alpha \geq 6$ and $k \geq 4$. Let $G$ be a graph of order $3 \alpha+4 k-3$ with $\delta(G) \geq 3 k-1$ and suppose $G$ contains no $k$ disjoint $\theta$-graphs. By induction on $k$, there exist $k-1$ disjoint $\theta$-graphs $T_{1}, T_{2}, \ldots T_{k-1}$ in $G$. Choose the $\theta$-graphs with $\left|T_{i}\right| \leq\left|T_{j}\right|$ for $i \leq j$, let $T=T_{1} \cup \cdots \cup T_{k-1}$ and suppose $|T|$ is minimized. Finally let $H=G \backslash T$. By induction on $k$, notice that $|H| \geq 4$.

Certainly $\left|T_{i}\right| \geq 5$ since, if $\left|T_{1}\right|=4$, we could remove $T_{1}$ and apply induction on $k$. First suppose $\left|T_{k-1}\right| \geq 6$. If $d_{T_{i}}(v) \geq 4$ for any $v \in H$ and $1 \leq i \leq k-1$, then $\left|T_{i}\right|$ can be made smaller using $v$. Similarly if $d_{T_{k-1}}(v) \geq 3$ then $\left|T_{k-1}\right|$ can be made smaller using $v$. This implies that for any vertex $v \in H, d_{T}(v) \leq 3(k-2)+2=3 k-4$ which implies that $\delta(H) \geq 3$. With $\delta(H) \geq 3$, there exists a a $\theta$-graph within $H$, completing the proof for this case.

Finally suppose $\left|T_{i}\right|=5$ for all $1 \leq i \leq k-1$. Again $d_{T_{i}}(v) \leq 3$ so $\delta(H) \geq 2$. If $\delta(H) \geq 3$ then again there exists a $\theta$-graph in $H$ so $\delta(H)=2$. Hence, $H$ is a collection of cycles joined by paths while avoiding a $\theta$-graph. In this graph, there exist an edge $x y$ such that $d_{H}(x), d_{H}(y)=2$. This implies that $e(\{x, y\}, T) \geq 6 k-2$. Therefore $e\left(\{x, y\}, T_{i}\right) \geq 5$ for some $i$, so $T_{i}$ can be made smaller using one or both of $x$ and $y$. This completes the proof of Theorem 16 . 
Acknowledgement: The authors would like to thank the Saturday Seminar group at Tokyo University of Science for their helpful comments and suggestions. In particular, we would like to thank Kenta Ozeki and Akira Saito for finding flaws in a previous draft.

\section{References}

[1] N. Alon. Disjoint directed cycles. J. Combin. Theory Ser. B, 68(2):167-178, 1996.

[2] D. Archdeacon and J. Šráň. Characterizing planarity using theta graphs. J. Graph Theory, 27(1):17-20, 1998.

[3] D. Bauer, H. J. Broersma, J. van den Heuvel, and H. J. Veldman. Long cycles in graphs with prescribed toughness and minimum degree. Discrete Math., 141(1-3):110, 1995.

[4] A. Bialostocki, D. Finkel, and A. Gyarfas. Disjoint chorded cycles in graphs. Discrete Mathematics - In Press, 2007.

[5] A. Blinco. Decompositions of complete graphs into theta graphs with fewer than ten edges. Util. Math., 64:197-212, 2003.

[6] H. L. Bodlaender. On disjoint cycles. In Graph-theoretic concepts in computer science (Fischbachau, 1991), volume 570 of Lecture Notes in Comput. Sci., pages 230-238. Springer, Berlin, 1992.

[7] B. Bollobás and G. Brightwell. Cycles through specified vertices. Combinatorica, 13(2):147-155, 1993.

[8] J. I. Brown, C. Hickman, A. D. Sokal, and D. G. Wagner. On the chromatic roots of generalized theta graphs. J. Combin. Theory Ser. B, 83(2):272-297, 2001.

[9] G. Chartrand and L. Lesniak. Graphs \& Digraphs. Chapman \& Hall/CRC, Boca Raton, FL, 4th edition, 2005.

[10] S. Chiba, S. Fujita, Y. Gao and G. Li. On a sharp degree sum condition for disjoint chorded cycles in graphs. Graph. Comb. 26:173-186, 2010.

[11] J. Chvatalova and J. Opatrný. The bandwidth of theta graphs. Utilitas Math., 33:9-22, 1988.

[12] Y. Egawa, H. Enomoto, S. Jendrol, K. Ota, and I. Schiermeyer. Independence number and vertex-disjoint cycles. Discrete Math., 307(11-12):1493-1498, 2007.

[13] Y. Egawa, R. J. Faudree, E. Györi, Y. Ishigami, R. H. Schelp, and H. Wang. Vertexdisjoint cycles containing specified edges. Graphs Combin., 16(1):81-92, 2000.

[14] Y. Egawa, S. Fujita, K. Kawarabayashi, and H. Wang. Existence of two disjoint long cycles in graphs. Discrete Math., 305(1-3):154-169, 2005.

[15] D. Eichhorn, D. Mubayi, K. O'Bryant, and D. B. West. The edge-bandwidth of theta graphs. J. Graph Theory, 35(2):89-98, 2000.

[16] H. Enomoto. On the existence of disjoint cycles in a graph. Combinatorica, 18(4):487492, 1998. 
[17] D. Finkel. On the number of independent chorded cycles in a graph. Discrete Math - In press, 2007.

[18] S. Fujita. Recent results on disjoint cycles in graphs. Electronic Notes in Discrete Mathematics, Volume 22, 7th International Colloquium on Graph Theory:409-412, 2005.

[19] S. Fujita. The generalized Ramsey numbers concerning three disjoint cycles and complete graphs. Submitted, 2008.

[20] A. J. Guttmann and S. G. Whittington. Two-dimensional lattice embeddings of connected graphs of cyclomatic index two. J. Phys. A., 11(4):721-729, 1978.

[21] L. Lovász. Combinatorial problems and exercises. North-Holland Publishing Co., Amsterdam, 1979.

[22] G. W. Peck and A. Shastri. Bandwidth of theta graphs with short paths. Discrete Math., 103(2):177-187, 1992.

[23] S. P. Radziszowski. Small Ramsey numbers. Electron. J. Combin., 1:Dynamic Survey $1,1994$.

[24] H. Wang. Large vertex-disjoint cycles in a bipartite graph. Graphs Combin., 16(3):359-366, 2000. 